\title{
Sawflies of the Keszthely Hills and its surroundings
}

\author{
AtTILA HARIS \\ H-1076 Budapest, Garay street 19 2/20, Hungary, e-mail: attilaharis@yahoo.com
}

HaRIs, A.: Sawflies of the Keszthely Hills and its surroundings.

Abstract: 173 species are reported from the Keszthely Hills and its surroundings. Rare species are Pamphilius jucundus (Eversmann, 1847); Pamphilius histrio Latreille, 1812; Orussus unicolor Latreille, 1812; Tremex alchymista Mocsáry, 1886; Tremex magus (Fabricius, 1787); Dolerus (Poodolerus) vernalis Ermolenko, 1964; Strongylogaster mixta (Klug, 1817); Fenella arenariae Zombori, 1978; Fenella nigrita Westwood, 1839; Periclista (Periclista) albiventris (Klug, 1816); Tenthredo (Cephaledo) neobesa Zombori, 1980; Euura fagi (Zaddach, 1876); Pristiphora (Pristiphora) albitibia (A. Costa, 1859) and Pristiphora (Pristiphora) fausta (Hartig, 1837).

Keywords: Hymenoptera, Symphyta, Keszthely Hills, Hungary, rare species

\section{Introduction}

Keszthely Hills is the westernmost member of the Transdanubian Mountains. It is built of dolomite, and enriched with limestone formations. The basalt formations of Kovácsi Hill and Tátika-region are the results of the volcanic activity. The other part of the investigated area is the coastal region of Lake Balaton. This region is mainly moorland and wet meadows which were originally part of the lake-bed and turned dry as a result of the water management in the 19th century.

The vegetation is abundant from the sub-Mediterranean xerotherm steppes, through closed oak and beech forests to cold gorges that preserve glacial remains. There are many habitat types with extremely rich flora and fauna of unique values.

No paper was published on the sawflies of this region so far, however, we may find good number of faunistic data on the series published by Dr. Lajos Zombori on the sawfly collection of the Natural History Museum of Zirc (Zombori 1973. 1979, 1980).

\section{Material and methods}

The studied and published material consists of 6 collection units. The main part comes from the collecting trips of the author in 2019. It contains 554 specimens of 95 species. Also the author collected sawflies around Keszthely during his university years between 1990 and 1992 . They are 38 species of 114 specimens. I identified the sawfly collection 
of the Zoological Department of Keszthely University. From this collection, 39 species of 56 specimens are published. The Sáringer collection, housed in Rippl-Rónai Museum, Kaposvár, contains from Keszthely approximately 40 specimens of 7 species. The author also partly identified and partly reidentified and checked the sawflies housed in the Hungarian Natural History Museum, Budapest. He found there 17 specimens of 14 species. Finally, he checked and reidentified the sawflies from this region deposited in the Natural History Museum of Zirc. They amount 92 specimens of 73 species.

In 2019, I spent 2 days in March, 11 days in April, only 3 days in May and 1 day in June on field. The rainfall made the collecting very difficult in May. The applied method was the net sweeping.

For identification, Zhelochovtsev's work on the sawflies of the European part of the former USSR (ZHELOCHOVTSEV 1988) was consulted. We also used some recent revisions and works to make the identifications even more precise (ACHTERBERG \& AARTSEN 1986, Blank \& Ritzau 1998, Haris 2001, 2006, Koch 1988, Zombori 2016).

For the discussion of the distribution of sawflies, we consulted the book of Roller and Haris titled Sawflies of the Carpathian Basin, History and Current Research (RoLLER \& HARIS 2008), the most recent European checklist of species (TAEGER et al. 2006) augmented by other faunistic records from the Carpathian Basin (MOCSÁRY 1900, ZOMBORI 1973, 1979, 1980, Roller 1993, 1994, 1996, 1998, 1999a, b, c, d, e, 2000, b, c, 2001, 2004, 2005, 2006a, b; 2010, Roller \& LukÁš 1999. Roller et al. 2006, Roller \& MACEK 2017, ROLLER \& OLŠOVSKÝ 2012, HARIS 2009, 2010, 2011, 2012, 2018a,b; HARIS \& GYURKOVICS 2012 ).

The higher classification of sawflies applied in this work follows the Hymenoptera part of Fauna Europaea (ACHTERBERG 2013).

\section{List of sites}

Only my collections from 1991-1992 and 2019 coordinated, the other parts of the material (Budapest, Zirc and Keszthely University collections) have no coordinates.

1991-1992

Keszthely, Újmajor: between $46^{\circ} 44^{\prime} 42.18^{\prime \prime} \mathrm{N}, 17^{\circ} 13^{\prime} 41.57^{\prime \prime} \mathrm{E}$ and $46^{\circ} 44^{\prime} 19.43^{\prime \prime} \mathrm{N}, 7^{\circ} 14^{\prime} 4.68^{\prime \prime} \mathrm{E}, 114-124 \mathrm{~m}$.

Keszthely: Szendrey-telep: forest belt: between $46^{\circ} 44^{\prime} 56.70^{\prime \prime} \mathrm{N}, 17^{\circ} 14^{\prime} 4.24^{\prime \prime} \mathrm{E}$ and $46^{\circ} 44^{\prime} 40.52^{\prime \prime} \mathrm{N}$, $17^{\circ} 14^{\prime} 12.43 " \mathrm{E}, 115-116 \mathrm{~m}$.

2019

Balatongyörök: Bélap-völgy, between 4646'27.16"N, 17²0'53.44"E and 46²47'4.61"N, 17²0'41.12"E, 168-196 m. Balatongyörök: Mogyorós út: Szőlőhegyek, between 46² $46^{\prime} 17.98^{\prime \prime} \mathrm{N}, 17^{\circ} 20^{\prime} 55.23^{\prime \prime} \mathrm{E}$ and $46^{\circ} 46^{\prime} 22.42^{\prime \prime} \mathrm{N}$, $17^{\circ} 20^{\prime} 56.65 " \mathrm{E}, 155-162 \mathrm{~m}$.

Cserszegtomaj: Büdöskúti út, between $46^{\circ} 48^{\prime} 29.04^{\prime \prime} \mathrm{N}, 17^{\circ} 16^{\prime} 14.33^{\prime \prime} \mathrm{E}$ and $46^{\circ} 48^{\prime} 27.62^{\prime \prime} \mathrm{N}, 17^{\circ} 16^{\prime} 32.55^{\prime \prime} \mathrm{E}$, 169-198 m.

Cserszegtomaj: Dobogó, between 46 $47^{\prime} 45.94^{\prime \prime} \mathrm{N}, 17^{\circ} 12^{\prime} 21.29^{\prime \prime} \mathrm{E}$ and $46^{\circ} 47^{\prime} 56.19^{\prime \prime} \mathrm{N}, 17^{\circ} 12^{\prime} 15.58^{\prime \prime} \mathrm{E}, 130-138 \mathrm{~m}$.

Cserszegtomaj: Fagyos-kereszt, between $46^{\circ} 50^{\prime} 29.67^{\prime \prime} \mathrm{N}, 17^{\circ} 16^{\prime} 3.41^{\prime \prime} \mathrm{E}$ and $46^{\circ} 50^{\prime} 34.25^{\prime \prime} \mathrm{N}, 1^{\circ} 16^{\prime} 2.10^{\prime \prime} \mathrm{E}$, 247-250 m.

Cserszegtomaj: Pilikáni dolomitbánya, between $46^{\circ} 47^{\prime} 43.78^{\prime \prime} \mathrm{N}, 1^{\circ} 15^{\prime} 58.17^{\prime \prime} \mathrm{E}$ and $46^{\circ} 47^{\prime} 39.07^{\prime \prime} \mathrm{N}$, $17^{\circ} 15^{\prime} 58.21$ "E 145-148 m.

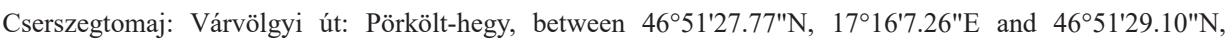
$17^{\circ} 15^{\prime} 52.07 " \mathrm{E}, 218-226 \mathrm{~m}$.

Fenékpuszta: régi vámház, between $46^{\circ} 42^{\prime} 35.26^{\prime \prime} \mathrm{N}, 17^{\circ} 14^{\prime} 53.46^{\prime \prime} \mathrm{E}$ and $46^{\circ} 42^{\prime} 39.20^{\prime \prime} \mathrm{N}, 17^{\circ} 14^{\prime} 55.73$ "E, 107-112 m. Gyenesdiás: Lőtér, between $46^{\circ} 47^{\prime} 6.96^{\prime \prime} \mathrm{N}, 1^{\circ} 17^{\prime} 42.40^{\prime \prime} \mathrm{E}$ and $46^{\circ} 47^{\prime} 10.13^{\prime \prime} \mathrm{N}, 1^{\circ} 17^{\prime} 46.74^{\prime \prime} \mathrm{E}, 188-208 \mathrm{~m}$. 


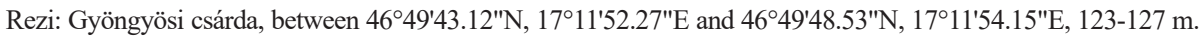

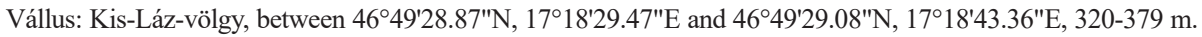
Vállus: Szarvas-kotrás, between $46^{\circ} 49^{\prime} 49.72^{\prime \prime} \mathrm{N}, 17^{\circ} 18^{\prime} 23.52^{\prime \prime} \mathrm{E}$ and $46^{\circ} 49^{\prime} 50.87^{\prime \prime} \mathrm{N}, 1^{\circ} 18^{\prime} 33.91^{\prime \prime} \mathrm{E}, 307-355 \mathrm{~m}$. Várvölgy: Bándi-mező, between 46 $52^{\prime} 18.23^{\prime \prime N}, 17^{\circ} 16^{\prime} 1.42^{\prime \prime} \mathrm{E}$ and $46^{\circ} 52^{\prime} 15.71^{\prime \prime} \mathrm{N}, 17^{\circ} 15^{\prime} 49.76^{\prime \prime} \mathrm{E}, 178-181 \mathrm{~m}$. Várvölgy: Zsidi-rét, between $46^{\circ} 51^{\prime} 58.31^{\prime \prime} \mathrm{N}, 1^{\circ} 17^{\prime} 35.72^{\prime \prime} \mathrm{E}$ and $46^{\circ} 51^{\prime} 45.38^{\prime \prime} \mathrm{N}, 1^{\circ} 17^{\prime} 24.98^{\prime \prime} \mathrm{E}, 183-193 \mathrm{~m}$. Vonyarcvashegy: Láprét, between $46^{\circ} 45^{\prime} 24.03^{\prime \prime} \mathrm{N}, 17^{\circ} 19^{\prime} 55.73^{\prime \prime} \mathrm{E}$ and $46^{\circ} 45^{\prime} 12.89^{\prime \prime} \mathrm{N}, 1^{\circ} 20^{\prime} 23.39^{\prime \prime} \mathrm{E}, 115-116 \mathrm{~m}$.

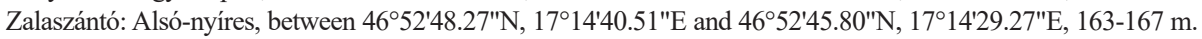

\section{Results}

\section{Xyelidae}

Xyela (Xyela) graeca J.P.E.F. Stein, 1876: Cserszegtomaj: Büdöskúti út, 16. 04. 2019, 1 female; Keszthely: Újmajor: Pine alley on Agrostis stolonifera ssp. gigantea field, 24. 04. 19911 female; Keszthely: Újmajor: on Trifolium incarnatum field. 22. 04. 1992, 1 male. Sporadic. Larva on Pinus nigra.

\section{Pamphiliidae}

Pamphilius alternans (Costa, 1860): Keszthely: Szendrey telep: forest belt. 04. 05. 1991,. 1 female. Sporadic. Hostplant unknown.

Pamphilius jucundus (Eversmann, 1847): Balatongyörök: Bélap-völgy, 19. 04. 2019,1 male. Rare. Hostplant uknown.

Cephalcia arvensis Panzer, 1805: Cserszegtomaj: Várvölgyi út: Pörkölt-hegy, 25. 05. 2019, 1 female. Sporadic. Hostplant: Picea spp.

Neurotoma nemoralis (Linné, 1758): Balatongyörök: Bélap-völgy, 22. 04. 2019, 2 females; Cserszegtomaj: Dobogó, 21. 04. 2019, 1 female; Cserszegtomaj: Büdöskúti út, 07. 04. 2019, 1 male. Frequent. Larva on Prunus mahaleb, P. armeniaca, P. spinosa and P. cerasus.

Pamphilius histrio Latreille, 1812: Keszthely, 24. 05. 2000, 1 female, 04. 2000, 1 female. Rare. Larva on Populus tremula.

Pamphilius betulae (Linné, 1758): Keszthely, 23. 05. 1992, 1 female. Sporadic. Hostplant: Populus tremula.

\section{Megalodontesidae}

Megalodontes plagiocephalus (Fabricius, 1804): Balatongyörök: Bélap-völgy, 19. 04. 2019, 1 female, 25. 05. 2019, 1 female; Cserszegtomaj: Dobogó, 11. 05. 2019, 1 male; Keszthely, 23. 05. 2000, 1 female. One of the most frequent Megalodontesidae species. Known hostplant: Peucedanum alsaticum.

\section{Orussidae}

Orussus abietinus (Scopoli, 1763): Cserszegtomaj: Várvölgyi street, 01. 05. 1991, 1 male. Sporadic. Parasitoid of Semanotus unduatus L.

Orussus unicolor Latreille, 1812: Vállus, 28. 05. 1964, 1 female.

\section{Xyphidriidae}

Xiphydria camelus (Linné, 1758): Keszthely, 05. 07. 1995, 1 female. Sporadic. Hostplants: Betula and Alnus spp.

Xiphydria longicollis (Geoffroy, 1785): Keszthely, 10. 05. 1992, 1 female. Sporadic. Hostplants: Acer, Quercus, Pinus and Betula spp. 


\section{Siricidae}

Tremex alchymista Mocsáry, 1886: Rezi: Cseres: Postaút, 12. 05. 2002, female. Rare. Hostplants: Quercus, Acer, Betula, Fagus and Carpinus spp.

Tremex fuscicornis (Fabricius, 1787): Keszthely, 20. 09. 1917, 1 female. The most frequent Siricidae. Hostplants: Fagus, Acer, Salix, Betula, Populus and Ulmus.

Tremex magus (Fabricius, 1787): Hévíz, 28. 05. 1967, 1 male. Rare. Hostplants: Betula, Quercus, Carpinus and Populus spp.

Urocerus gigas (Linné, 1758): Vállus: Büdöskút, 27. 10. 1964, 2 females. Sporadic. Larva lives in Pinus, Picea, Larix and Abies spp. but also reared from Populus and Fraxinus spp. (based on very old dubious records).

\section{Cephidae}

Calameuta (Calameuta) filiformis (Eversmann, 1847): Várvölgy: Zsidi-rét, 25. 05. 2019, 1 female; Keszthely Hills, 29. 05. 1957, 1 female. Generally common species. Larva lives in stems of Arrhenaterum elatius, Phalaris arundinacea, Calamagrostis epigeios, Elytrigia repens and Phragmites communis.

Calameuta (Calameuta) haemorrhoidalis (Fabricius, 1781): Vállus: Barbacs, 22. 05. 1966, 1 female. Frequent. Hostplant unknown.

Calameuta (Calameuta) pallipes (Klug, 1803): Zalaszántó: Kovácsi-hegy, 01-02. 05. 1959, 1 male; Vállus: Láz-tető: 28. 05. 1964, 1 female. Frequent on diverse Poaceae.

Cephus brachycercus Thomson, 1871: Balatongyörök: Bélap-völgy, 27. 04. 2019, 1 female; Keszthely: Camping, 08. 05. 1991. 1 female. Widely distributed, sporadic species. Hostplant unknown.

Cephus pygmeus (Linné, 1767): Balatongyörök: Mogyorós út: Szőlöhegyek, 16. 04. 2019, 1 female; Cserszegtomaj: Dobogó, 18. 05. 2019, 1 female; Keszthely, 21. 05. 2002, 1 female. Common. Insect pest of cereals and Gramineae.

Cephus spinipes (Panzer, 1800): Balatongyörök: Bélap-völgy, 25. 05. 2019, 1 female, 1 male, 01. 06. 2019, 1 female; Cserszegtomaj: Várvölgyi út: Pörkölt-hegy, 25. 05. 2019, 1 male; Cserszegtomaj: Dobogó, 11. 05. 2019, 1 male. Frequent. Hostplant: Phleum pratense.

Hartigia nigra (Harris, 1776): Balatongyörök: Bélap-völgy, 11. 05. 2019, 1 male; Zalaszántó: Kovácsi-hegy, 01-02. 05. 1959, 1 female. Sporadic in Hungary. Hostplants: Rosa and Rubus spp.

Janus compressus (Fabricius, 1793): Keszthely, May 1986,. 1 male. Frequent insect pest of Pyrus and Malus spp.

Janus luteipes (Lepeletier, 1823): Keszthely, 27. 08. 1980, 1 female. Rare. Hostplants: Salix, Populus and Viburnum spp.

Trachelus troglodyta (Fabricius, 1787): Vállus: Büdöskút: Fekete-hegy, 26. 05. 1964, 1 female. Sporadic. Hostplant: Secale cereale.

\section{Argidae}

Arge cyanocrocea (Förster, 1771): Balatongyörök: Bélap-völgy, 25. 05. 2019, 1 female, 18. 05. 2019, 1 female; Cserszegtomaj: Dobogó, 25. 05. 2019, 1 female, 11. 05. 2019, 1 female, 11. 05. 2019, 2 males; Cserszegtomaj: Várvölgyi út: Pörkölt-hegy, 01. 06. 2019, 1 female; Keszthely, 03. 02. 1992, 1 female, 17. 05, 1992, 1 female, 01. 05. 2991, 1 female. Common species. Known hostplants: Rubus idaeus and Sanguisorba officinalis.

Arge berberidis Schrank, 1802: Balatongyörök: Bélap-völgy, 18. 04. 2019, 1 female, 22. 04. 2019, 1 female, 25. 05. 2019, 1 male; Cserszegtomaj: Dobogó, 21. 04. 2019, 1 female, 23. 04. 2019, 1 male. Frequent. Larva on Berberis and Mahonia spp. 


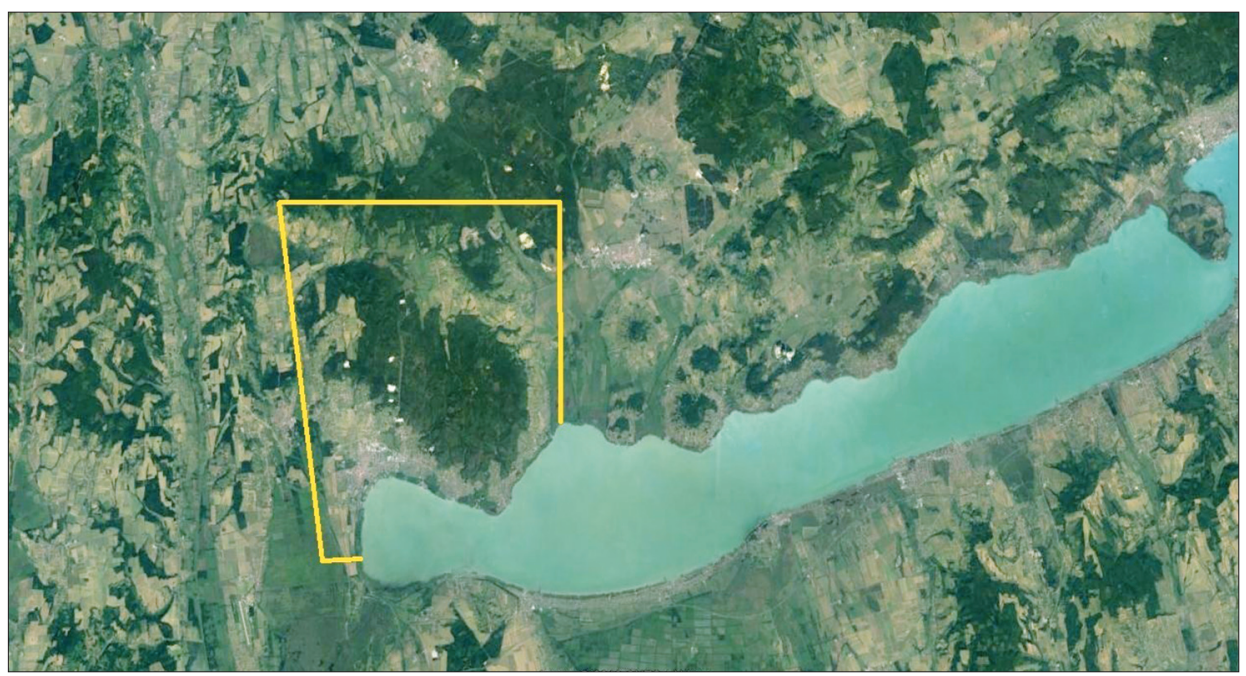

Fig. 1. The investigated area at Lake Balaton

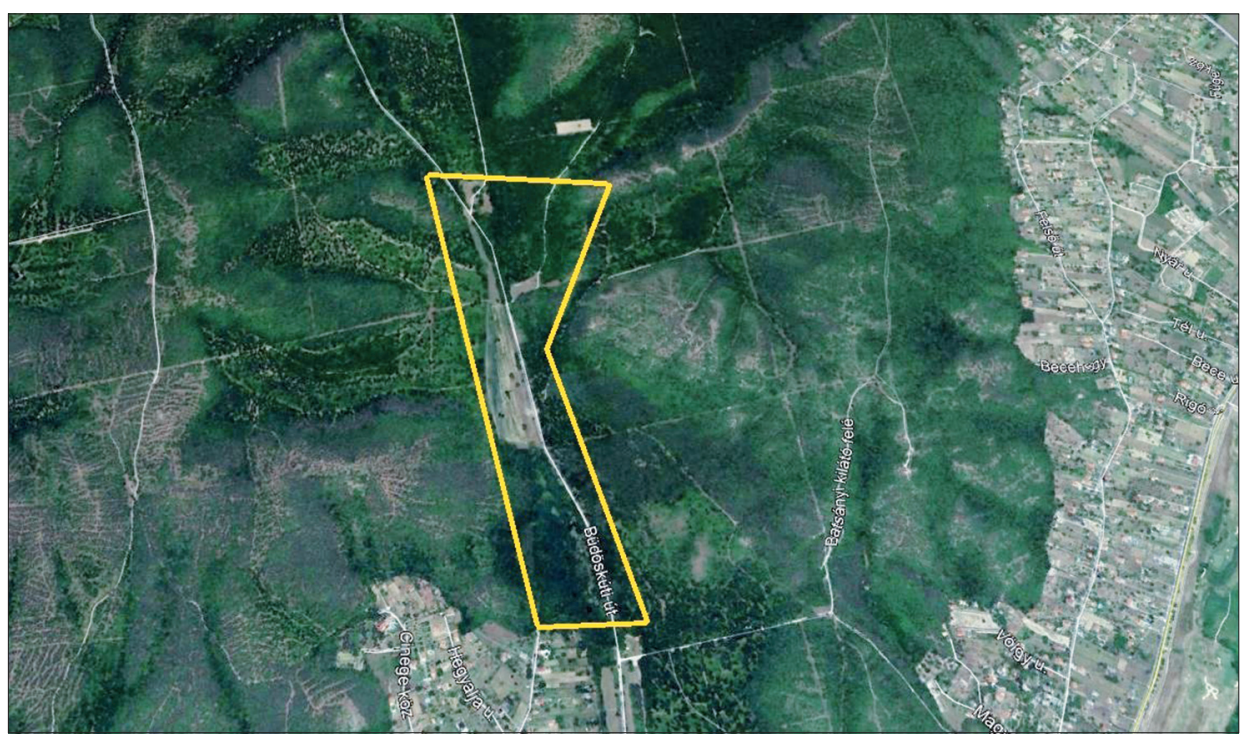

Fig 2. Map of Bélap völgy (Bélap Valley)

Arge enodis (Linné, 1767): Várvölgy, 23. 08. 1979, 1 female.; 21. 07. 1972, 1 female. Frequent. Larva on smooth leaved Salix spp.

Arge gracilicornis (Klug, 1814): Zalaszántó: Tátika-erdő, 13. 08. 1966, 3 females. Sporadic. Larva on Rubus idaeus.

Arge melanochra (Gmelin, 1790): Balatongyörök: Bélap-völgy, 25. 05. 2019, 1 female, 01. 06. 2019, 2 females; Várvölgy: Bándi-mező, 01. 06. 2019, 3 females, 4 males; Vállus: Láz-tető, 28. 05. 1964, 1 male. Frequent, the most common Argid sawfly. Hostplant: Crataegus oxycantha. 


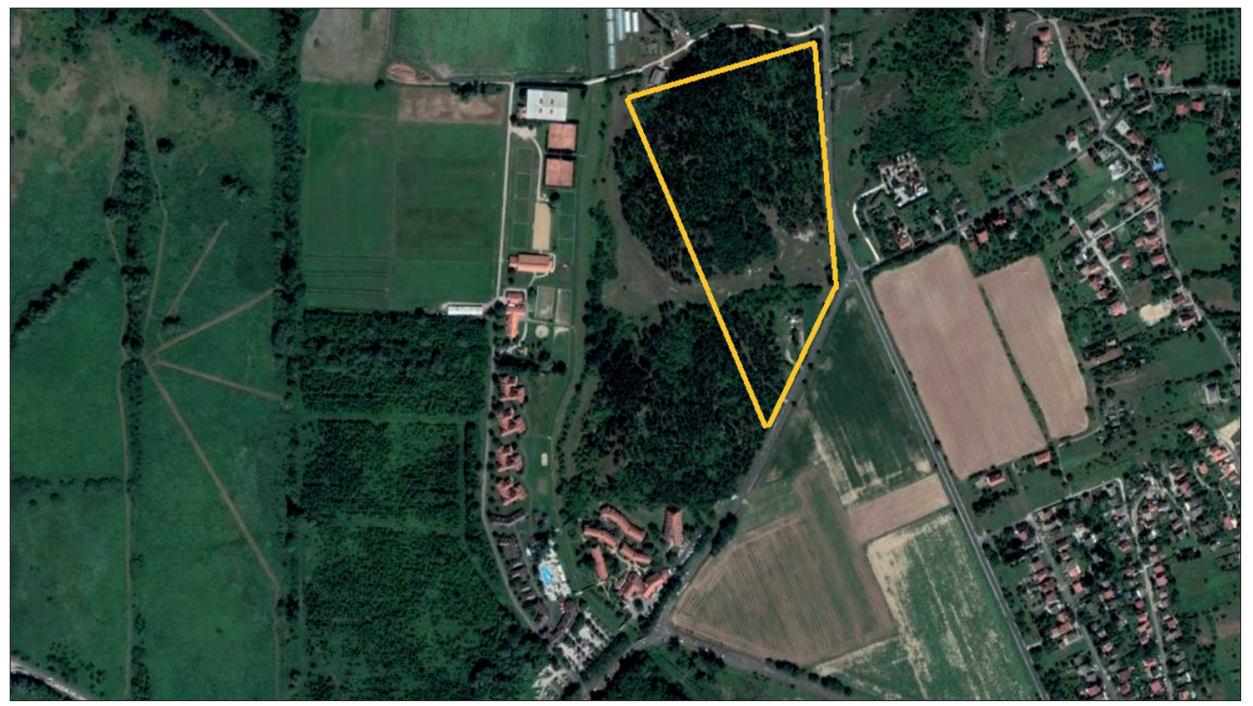

Fig. 3. Map of Dobogó

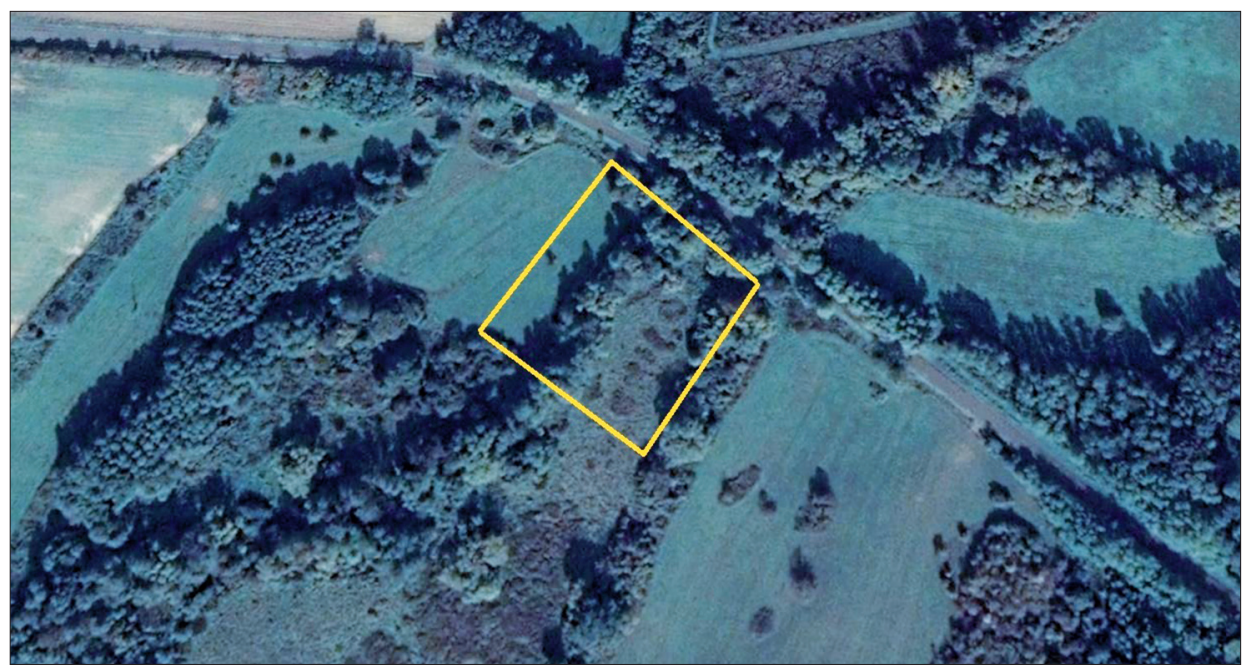

Fig. 4. Map of Zalaszántó: Alsó-nyíres

Arge nigripes (Retzius, 1783): Balatongyörök: Bélap-völgy, 22. 04. 2019, 1 female. Sporadic. Hostplants: Rosa spp.

Arge ochropus (Gmelin, 1790): Zalaszántó: Tátika-erdő, 13. 08. 1966, 1 female. Locally frequent. Pest of Rosa spp.

Arge pagana ssp. pagana (Panzer, 1797): Cserszegtomaj: Fagyos-kereszt, 01. 06. 2019, 1 male; Keszthely, 10. 05. 2000, 1 male; Zalaszántó: Tátika-erdő, 13. 08. 1966, 1 female. Frequent. Hostplants: Rosa spp.

Arge rustica (Linné, 1758): Balatongyörök: Bélap-völgy, 01. 06. 2019, 2 females; Vállus: Láz-tető, 28. 05. 1964, 1 female. Sporadic. Hostplant: Quercus spp. 


\section{Cimbicidae}

Abia aenea (Klug, 1820): Balatongyörök, 02. 07. 1978, 1 female. Sporadic. Hostplants: Lonicera and Symphoricarpos spp.

Abia sericea (Linné, 1758): Zalaszántó: Tátika-erdő, 13. 08. 1966, 1 female, 1 male. Frequent. Larva on Succisa, Knautia and Fragaria spp.

Cimbex femoratus (Linné, 1758): Keszthely, 11. 05. 1986, 1 male, 22. 05. 1997, 1 male. Widely distributed, frequent species. Hostplants: Betula pendula and B. pubescens.

Cimbex luteus (Linné, 1758): Keszthely, 20. 05. 2017, 1 female. Sporadic; Vállus: Szentmiklósi-völgy, 22. 05. 1966, 1 female. Larva on Populus tremula and Salix spp.

Cimbex quadrimaculatus (O.F. Müller, 1766): Keszthely, 11. 05. 1994, 1 female. Frequent insect pest. Larva on Crataegus, Pyrus and Prunus spp.

Corynis crassicornis (Rossi, 1790): Vállus, 20-21. 05. 1969, 1 female. Sporadic. Hosts are Sedum spp. (S. album, S. acre and S. sexangulare).

Trichiosoma tibiale Stephens, 1835: Keszthely, 01. 05. 2000, 1 female. Rare. Larva on Crataegus (need confirmation), Spiraea, Sorbus aucuparia and Salix spp.

\section{Diprionidae}

Diprion pini (Linné, 1758): Keszthely, 20. 06. 1990, 1 male. Frequent. Insect pest of Pinus spp.

\section{Tenthredinidae \\ Dolerinae}

Dolerus (Achaetoprion) triplicatus (Klug, 1818): Keszthely, 01. 05. 2002, 1 male. Sporadic. Larva on Juncus filiformis and J. effusus.

Dolerus (Dicrodolerus) vestigialis (Klug, 1818): Balatongyörök: Bélap-völgy, 22. 04. 2019, 1 female, 19. 04. 2019, 1 female, 21. 04. 2019, 1 female, 20. 04. 2019, 1 male; Vonyarcvashegy: Láprét, 18. 05. 2019, 1 female, 21. 04. 2019, 1 male, 27. 04. 2019, 1 male; Vállus: Szentmiklósi-völgy, 22. 05. 1966, 1 female. Common. Hostplants: Equisetum palustre, E. sylvaticum, E. arvense and E. pratense.

Dolerus (Oncodolerus) eversmanni W. F. Kirby, 1882: Várvölgy: Zsidi-rét, 17. 04. 2019, 2 males. Frequent. Larva on Equisetum arvense and E. palustre.

Dolerus (Poodolerus) aeneus Hartig, 1837: Cserszegtomaj: Dobogó, 17. 04. 2019, 1 male; Cserszegtomaj: Keszthely Hills: Pilikáni street, 22. 05. 1991, 1 female. Sporadic. Hostplants: Graminae.

Dolerus (Dolerus) aericeps Thomson, 1871: Várvölgy, 21. 07. 1972, 1 female; Zalaszántó: Kovácsi-hegy, 14. 08. 1966, 4 males. Frequent. Larva on Equisetum palustre.

Dolerus (Dolerus) germanicus ssp. germanicus (Fabricius, 1775): Vonyarcvashegy: Láprét, 27. 04. 2019, 1 female; Várvölgy: Zsidi-rét, 17. 04. 2019, 1 male; Keszthely: Szendrey telep, 01. 05. 1991, 1 male; Zalaszántó: Kovácsi-hegy, 14. 08. 1966, 1 female. Common. Larva on Equisetum arvense and E. palustre.

Dolerus (Poodolerus) asper Zaddach, 1859: Balatongyörök: Bélap-völgy, 23. 04. 2019, 1 female. Sporadic. Hostplants: Graminae and Cyperaceae.

Dolerus (Poodolerus) brevicornis Zaddach, 1859: Rezi: Gyöngyösi csárda, 06. 04. 2019, 1 female; Zalaszántó: Alsó-nyíres, 20. 04. 2019, 1 female. Sporadic. Larva on Graminae.

Dolerus (Poodolerus) gonager (Fabricius, 1781): Vállus: Szarvas-kotrás, 07. 04. 2019, 1 female; Vállus: Kis-Láz-völgy, 17. 04. 2019, 1 female; Balatongyörök: Bélapvölgy, 23. 04. 2019, 1 female; Fenékpuszta: régi vámház, 30. 03. 2019, 1 male; Keszthely: Újmajor on Triticum aestivum 02. 04. 1990, 1 female; Keszthely: Újmajor on 
Agrostis stolonifera ssp. gigantea 03. 04. 1991, 4 males, 07. 04. 1991, 1 male, 09. 04. 1991, 1 male. Common. Larva on Graminae.

Dolerus (Poodolerus) haematodes (Schrank, 1781): Keszthely: Újmajor on Agrostis s. ssp. g. 21. 03. 1991, 1 female; Keszthely: Újmajor on Festuca rubra 03. 04. 1991, 1 male. Frequent. Larva on Juncus, Scirpus, Crex and Gramineae.

Dolerus (Poodolerus) liogaster C. G. Thomson, 1871: Cserszegtomaj: Várvölgyi út: Pörkölt-hegy, 07. 04. 2019, 1 female.

Dolerus (Poodolerus) nigratus (O.F. Müller, 1776): Fenékpuszta: Régi vámház, 30. 03. 2019, 1 female; Zalaszántó: Alsó-nyíres, 31. 03. 2019, 2 females, 20. 04. 2019, 2 females; Cserszegtomaj: Fagyos-kereszt, 17. 04. 2019, 1 female, 1 male; Rezi: Gyöngyösi csárda, 17. 04. 2019, 1 female; Balatongyörök: Bélap-völgy, 20. 04. 2019, 1 female, 21. 04. 2019, 1 female; Várvölgy: Zsidi-rét, 30. 03. 2019, 1 male; Várvölgy: Bándi-mezö, 07. 04. 2019, 1 male; Keszthely: Újmajor on Agrostis s. ssp. gigantea. 16. 03. 1990, 1 female; Keszthely: Újmajor on Triticum aestivum 16. 03. 1990, 1 male; Keszthely: Újmajor on Agrostis s. ssp. g. 24. 04. 1991, 1 female; Keszthely: Újmajor on Festuca rubra 29. 04. 1991, 1 female; Keszthely: Úmajor on Agrostis s. ssp. g. 29. 04. 1991, 1 female; 25. 04. 1990, 1 female, 03. 04. 1991, 1 female; Keszthely: Újmajor on Triticum aestivum 03. 04. 1991, 3 females, Keszthely: Újmajor on Agrostis s. ssp. g. 07. 04. 1991, 1 female; Keszthely on Triticum aestivum 24. 04. 1991, 2 females; Keszthely: Újmajor on Agrostis s. ssp. g. 09. 04. 1991, 1 female; Keszthely: Újmajor on Triticum aestivum 09. 04. 1991, 1 female; Keszthely: Újmajor on Agrostis s. ssp. g. 16. 04. 1991, 1 female; Keszthely: Szendrey telep on flower of Cerasus avium, 21. 04. 1992, 1 female; Keszthely: Újmajor on Agrostis s. ssp. g. 03. 04. 1991, 7 males; Keszthely: Újmajor on Agrostis s. ssp. g. 07. 04. 1991, 8 males; Keszthely: Úmajor on Agrostis s. ssp. g. 09. 04. 1991, 6 males, 16. 04. 1991, 3 males; Keszthely: Újmajor on Agrostis s. ssp. g. 16. 03. 1990, 1 male; Keszthely: Újmajor on Agrostis s. ssp. g. 02. 04. 1990, 1 male, 13. 04. 1990, 1 male, 21. 03. 1991, 1 male; Várvölgy: Nagyház-tető, 21. 05. 1969, 1 female. Common. Larva on Gramineae including cereals.

Dolerus (Poodolerus) nitens Zaddach, 1859: Várvölgy: Zsidi-rét, 07. 04. 2019, 1 male; Zalaszántó: Kovácsi-hegy, 02-03. 05. 1959, 1 male.. Sporadic. Larva on Cyperaceae and Graminae.

Dolerus (Poodolerus) picipes (Klug, 1818): Balatongyörök: Bélap-völgy, 19. 04. 2019, 1 female; Keszthely: Úmajor on Agrostis s. ssp. g. 13. 04. 1990, 1 female; Keszthely: Szendrey telep on Triticum aestivum 16. 04. 1991, 1 female; Keszthely on Secale cereale 11. 04. 1989, 1 female. Frequent. Larva on Graminae.

Dolerus (Poodolerus) puncticollis Thomson, 1871: Balatongyörök: Bélap-völgy, 06. 04. 2019, 2 females, 22. 04. 2019, 1 female; Várvölgy: Bándi-mező, 07. 04. 2019, 1 female; Várvölgy: Zsidi-rét, 07. 04. 2019, 1 female; Rezi: Gyöngyösi csárda, 17. 04. 2019, 1 female; Keszthely: Újmajor on Agrostis s. ssp. g. 03. 04. 1991, 1 male, 1 female; Keszthely: Újmajor on Triticum aestivum 03. 04. 1991, 1 male, 6 females;, Keszthely: Újmajor on Festuca rubra 03. 04. 1991, 1 female; Keszthely: Újmajor on Festuca pseudovina, 21. 03. 1990, 1 female; Keszthely: Újmajor on Agrostis s. ssp. g. 07. 04. 1991, 2 females; Keszthely: Úmajor on Triticum aestivum 07. 04. 1991, 2 females; Keszthely: Újmajor on Agrostis s. ssp. g., 09. 04. 1991, 1 female, 1990. 03. 21.1 female; Keszthely Újmajor Triticum aestivum 1990. 03. 21. 1 female; Keszthely Újmajor grass culture, 1991. 04. 03. 1 female; Keszthely: Szendrey telep on Triticum aestivum 24. 04. 1991, 1 female; Keszthely: Szendrey telep on flower of Malus domestica, 14. 04. 1992, 2 females. Common. Larva on Graminae including cereals.

Dolerus (Poodolerus) sanguinicollis (Klug, 1818): Keszthely: Park next to Halászcsárda, 08. 05. 1991, 1 female. Sporadic. 
Dolerus (Poodolerus) vernalis Ermolenko, 1964: Keszthely, 04. 04. 1974, 1 female. Rare. Hostplant unknown.

\section{Allantinae}

Allantus (Emphytus) didymus (Klug, 1818): Balatongyörök: Bélap-völgy, 18. 04. 2019, 1 male. Sporadic. Larva on Sanguisorba minor: old records from Rubus and Rosa spp. need checking.

Allantus (Emphytus) cingulatus (Scopoli, 1763): Balatongyörök: Bélap-völgy, 27. 04. 2019, 1 female, 1 male; Várvölgy: Nagyláz-tető, 21. 05. 1969, 1 female. Frequent. Larva on Fragaria and Rosa spp.

Allantus (Emphytus) cinctus (Linné, 1758): Balatongyörök: Mogyorós út: Szőlőhegyek, 18. 04. 2019, 1 female; Balatongyörök: Bélap-völgy, 20. 04. 2019, 1 male. Frequent. Hostplants: Rosa spp.

Allantus (Emphytus) calceatus (Klug, 1818): Várvölgy: Zsidi-rét, 17. 04. 2019, 1 female; Balatongyörök: Bélap-völgy, 23. 04. 2019, 1 female. Sporadic. Hostplants: Rubus, Sanguisorba, Rosa, Filipendula, Fragaria and Alchemilla spp

Allantus (Emphytus) melanarius (Klug, 1818): Balatongyörök: Bélap-völgy, 22. 04. 2019, 1 female. Frequent. Hostplant: Cornus sanguinea.

Ametastegia (Protemphytus) carpini (Hartig, 1837): Balatongyörök: Bélap-völgy, 23. 04. 2019, 1 female, 20. 04. 2019, 1 female, 18. 04. 2019, 1 female. Sporadic. Hostplant: Geranium spp.

Ametastegia (Ametastegia) equiseti (Fallén, 1808): Keszthely, 08. 05. 1991, 1 female.

Frequent. Larva on Chenopodium album, Lythrum salicaria, Polygonum persicaria and Rumex acetosella.

Ametastegia (Ametastegia) glabrata (Fallén, 1808): Keszthely 20-30. 05. 1992, 1 female. Frequent. Larva on Chenopodiaceae, Polygonaceae, Plantago, Salix, Lithrum, Ribes and Rubus spp.

Athalia ancilla ssp. ancilla Serville, 1823: Keszthely, 13. 06. 1957, 1 female, 03. 09. 1955, 1 female, 16. 06. 1958, 1 female, 31. 05. 1957, 1 female, 31. 05. 1956, 1 female, 17. 06. 1957,1 male, 24. 08. 1957, 1 male, 05. 09. 1957, 1 male, 06. 09. 1954, 1 male, 27. 06. 1954, 1 male, 04. 07. 1957, 1 female, 07. 08. 1957, 1 female, 27. 06. 1957, 1 female. Insect pest. Hostplants: Diplotaxis tenuifolia, Erysimum cheirnthoides, Raphanus raphanistrum, Sinapsis spp.

Athalia bicolor Serville, 1823: Balatongyörök: Bélap-völgy, 11. 05. 2019, 1 male; Várvölgy: Zsidi-rét, 25. 05. 2019, 1 male; Keszthely, 04. 06. 1954, 1 female, 31. 05. 1956, 1 female, 29. 05. 1957, 1 female. Frequent. Hostplant: Ranunculus spp.

Athalia circularis (Klug, 1815): Várvölgy: Bándi-mezö, 11. 05. 2019, 1 female; Keszthely 20. 06. 1990, 1 female. Frequent. Hostplants: Arctium lappa, Ajuga reptans, Veronica beccabunga, V. longifolia, V. officinalis, Alliaria petiolata, Glechoma hederacea, Melampyrum, Capsella and Lycopus spp.

Athalia cordata Serville, 1823: Balatongyörök: Bélap-völgy, 19. 04. 2019, 1 female, 2 males, 22. 04. 2019, 1 female, 16. 04. 2019, 2 females, 6 males, 18. 04. 2019, 1 male, 21. 04. 2019, 1 male; Balatongyörök: Mogyorós út: Szőlőhegyek, 16. 04. 2019, 4 males; Cserszegtomaj: Dobogó, 21. 04. 2019, 1 male; Cserszegtomaj: Fagyos-kereszt, 20. 04. 2019, 1 female; 17. 04. 2019, 1 male. Keszthely 19. 10. 1991, 1 male; Keszthely: University Dorm. Park 02. 05. 1991, 1 male; Keszthely: Szendrey telep: forest belt, 14. 05. 1991, 1 male, 1 female, 21. 05. 1991, 1 male; Keszthely: Újmajor: on Medicago sativa field, 11. 09. 1992, 1 female; Cserszeg, 04. 07. 1992, 1 female; Keszthely, 22. 05. 2002, 1 female, 12. 05. 2002, 1 female, 11. 04. 1998, 1 female; Zalaszántó: Kovácsihegy, 02-03. 05. 1959, 3 males, Zalaszántó: Tátika-erdő, 13. 08. 1966, 1 female, Vállus: 
Csetény, 23. 05. 1969, 1 female. Common. Larva on Misopates orontinum, Antirrhinum majus, Ajuga reptans, Teucrium scorodonia and Plantago spp.

Athalia liberta (Klug, 1815): Vállus: Láz-tető, 28. 05. 1964, 1 female. Frequent. Feeding on Alliaria petiolata, Arabidopsis thaliana, Cardamine hirsuta and Sisymbrium officinale.

Athalia rosae (Linné, 1758): Balatongyörök: Bélap-völgy, 18. 04. 2019, 2 females, 21. 04. 2019, 1 female, 22. 04. 2019, 1 female, 23. 04. 2019, 2 females; Cserszegtomaj: Dobogó, 21. 04. 2019, 1 female; Vonyarcvashegy: Láprét, 21. 04. 2019, 1 female; Zalaszántó: Alsó-nyíres, 11. 05. 2019, 1 female; Cserszeg 20-21. 06. 1992, 1 female; Keszthely, 21. 05. 2017, 1 female; Hévíz, 17. 09. 2016, 1 female. Common pest. Hostplants: Raphanus sativus, $R$. raphanistrum, Sinapis arvensis, Sisymbrium officinale, Armoracia rusticana, Barbarea sp., Brassica napus, B. juncea, B. rapa, B. oleracea, Tropaeolum majus, Sinapis arvensis, Alliara petiolata and Cardamine spp.

Empria liturata (Gmelin, 1790): Balatongyörök: Bélap-völgy, 23. 04. 2019, 1 female, 22. 04. 2019, 1 female, 20. 04. 2019, 1 female, 18. 04. 2019, 1 female, 21. 04. 2019, 1 female, 1 male, 21. 04. 2019, 1 female, 18. 04. 2019, 1 female, 20. 04. 2019, 1 female, 16. 04. 2019, 1 female, 1 male; Balatongyörök: Mogyorós út: Szőlőhegyek, 18. 04. 2019, 1 female, 06. 04. 2019, 1 male, 16. 04. 2019, 1 male; Cserszegtomaj: Büdöskúti út, 07. 04. 2019, 1 male, 16. 04. 2019, 1 male; Cserszegtomaj: Dobogó, 22. 04. 2019, 1 male; Cserszegtomaj: Fagyos-kereszt, 17. 04. 2019, 1 male, 20. 04. 2019, 1 male; Várvölgy: Bándi-mező, 31. 03. 2019, 1 male; Vállus: Kis-Láz-völgy, 17. 04. 2019, 1 female. Frequent. Hostplants: Fragaria and Geum spp.

Empria parvula (Konow, 1892): Balatongyörök: Bélap-völgy, 23. 04. 2019, 1 female, 21. 04. 2019, 1 female, 16. 04. 2019, 1 female. Sporadic. Hostplant unknown.

Empria sexpunctata (Serville, 1823): Cserszegtomaj: Büdöskúti út, 07. 04. 2019, 1 female, 16. 04. 2019, 1 female; Balatongyörök: Bélap-völgy, 20. 04. 2019, 1 female, 11. 05. 2019, 1 female. Frequent. Larva on Geum spp.

Empria tridens (Konow, 1896): Cserszegtomaj: Dobogó, 22. 04. 2019, 1 female; Balatongyörök: Bélap-völgy, 27. 04. 2019, 1 female. Frequent. Hostplants: Geum spp. and Rubus idaeus.

Monostegia abdominalis (Fabricius, 1798): Keszthely, 01. 08. 1959, 1 female; Zalaszántó: Tátika-erdő, 13. 08. 1966, 4 females. Fequent. Recorded on Glaux maritima, Lysimachia numularia and L. vulgaris.

Monostegia cingulata (Konow, 1891): Zalaszántó: Kovácsi-hegy,28. 06. 1965, 1 female; Vállus: Büdöskút: Fekete-hegy, 26. 05. 1964, 6 females.

Taxonus agrorum (Fallén, 1808): Balatongyörök: Bélap-völgy, 23. 04. 2019, 1 female, 27. 04. 2019, 1 female; Keszthely, 20. 10. 1998, 1 male. Frequent. Hostplants: Rubus idaeus and $R$. caesius.

Taxonus sticticus (Klug, 1817): Vállus: Büdöskút: Fekete-hegy, 26. 05. 1964,1 female. Sporadic. Hostplant unknown.

\section{Selandrinae}

Birka cinereipes (Klug, 1816): Cserszegtomaj: Dobogó, 22. 04. 2019, 1 female; Balatongyörök: Bélap-völgy, 27. 04. 2019. Sporadic. Hostplants: Myosotis spp.

Nesoselandria morio (Fabricius, 1781): Balatongyörök: Bélap-völgy, 11. 05. 2019, 1 female; Keszthely, 30. 05. 1960, 1 female, 06. 07. 1960, 1 female, 08. 10. 1960, 1 female, 29. 05. 1958, 1 female. Frequent. Hostplants: Brachytecium reflexum, Ceratodon purpureus, Chenopodium album, Dicranum scoparium, Fragaria vesca, Hedwigia ciliata, Myosotis arvensis, Plagiomnium cuspidatum, Plagiothecium denticulatum, 
Polygonum aviculare, Polytrichum commune, Pseudobryum cinclidiodes, Sanionia uncinata, Stellaria media, Veronica chamaedrys and $V$. officinalis.

Selandria serva (Fabricius, 1793): Keszthely: Szendrey telep: forest belt, 21. 05. 1991, 1 male. Frequent. Host plants: Poaceae, Carex spp. and Juncus spp.

Strongylogaster mixta (Klug, 1817): Keszthely, 25. 04. 2007, 1 female. Rare. Hostplants: Athyrium filix-femina, Pteridium aquilinum and Aspidium spp.

\section{Heterarthrinae}

Endelomyia aethiops (Gmelin, 1790): Balatongyörök: Bélap-völgy, 22. 04. 2019, 1 female. Sporadic. Larva on Rosa spp.

Fenella arenariae Zombori, 1978: Keszthely, 27. 07. 1977, 1 female. Rare. Larva on Potentilla arenaria.

Fenella nigrita Westwood, 1839: Keszthely: Camping, 30. 06. 1982, 1 female. Rare. Hostplants: Potentila reptans and Agrimonia eupatoria.

Hinatara nigripes (Konow, 1907): Balatongyörök: Bélap-völgy, 16. 04. 2019, 1 female; Cserszegtomaj: Fagyos-kereszt, 17. 04. 2019, 1 female. Sporadic. Hostplant: Acer capestre.

\section{Blennocampinae}

Blennocampa phyllocolpa Viitasaari \& Vikberg, 1985: Keszthely: Szendrey telep, April 1993, 1 male. Frequent. Larva rolls the leaves of Rosa spp.

Eutomostethus ephippium (Panzer, 1798): Zalaszántó: Alsó-nyíres, 11. 05. 2019, 1 male; Vonyarcvashegy: Láprét, 21. 04. 2019, 1 female; Cserszegtomaj: Várvölgyi út: Pörkölt-hegy, 01. 06. 2019, 1 female. Common, larva on Poaceae.

Eutomostethus gagathinus (Klug, 1816): Vonyarcvashegy: Láprét, 27. 04. 2019, 1 female. Sporadic. Hostplant unknown.

Eutomostethus luteiventris (Klug, 1816): Balatongyörök: Bélap-völgy, 19. 04. 2019, 1. female; Keszthely: Szendrey telep 01. 05. 1991, 1 female. Locally frequent. Larva on Juncus effusus.

Claremontia alternipes (Klug, 1816): Balatongyörök: Bélap-völgy, 18. 04. 2019, 1 female; Vállus: Kis-Láz-völgy, 17. 04. 2019, 1 female. Sporadic. Hostplant: Rubus idaeus.

Claremontia brevicornis (Brischke, 1883): Várvölgy: Bándi-mező, 30. 03. 2019, 1 female; Balatongyörök: Bélap-völgy, 20. 04. 2019, 1 female, 18. 04. 2019, 1 female, 22. 04. 2019, 1 female; Cserszegtomaj: Fagyos-kereszt, 17. 04. 2019, 1 female; Zalaszántó: Alsó-nyíres, 16. 04. 2019, 2 males.

Claremontia waldheimii (Gimmerthal, 1847): Balatongyörök: Bélap-völgy, 16. 04. 2019, 1 female; Balatongyörök: Mogyorós út: Szőlőhegyek, 18. 04. 2019, 1 female. Frequent. Hostplant: Geum urbanum.

Halidamia affinis (Fallén, 1807): Keszthely: Szendrey telep 08. 05. 1991, 1 female. Frequent. Hostplants: Galium aparine and G. molugo.

Monophadnoides rubi (Harris, 1845): Rezi: Gyöngyösi csárda, 06. 04. 2019, 1 female; Zalaszántó: Alsó-nyíres, 20. 04. 2019, 1 female. Frequent. Hostplant: Rubus fructicosus.

Monophadnus pallescens (Gmelin, 1790): Rezi: Gyöngyösi csárda, 31. 03. 2019, 1 female; Cserszegtomaj: Várvölgyi út: Pörkölt-hegy, 07. 04.2019, 1 female; Cserszegtomaj: Dobogó, 17. 04. 2019, 1 female; Balatongyörök: Mogyorós út: Szőlőhegyek, 16. 04. 2019, 1 female; Balatongyörök: Bélap-völgy, 20. 04. 2019, 1 female, 22. 04. 2019, 1 female, 23. 04. 2019, 1 female; Keszthely, 06. 04. 1957, 1 female. Common. Hostplants: Ranunculus acris, $R$. repens, $R$. lanuginosus and Anemone nemorosa. 
Monophadnus spinolae (Klug, 1816): Balatongyörök: Bélap-völgy, 18. 05. 2019, 1 female; Vállus: Láz-tető: 28. 05. 1964, 2 males. Sporadic. Hostplants: Clematis vitalba and C. flammula.

Pareophora pruni (Linné, 1758): Cserszegtomaj: Dobogó, 21. 04. 2019, 1 female, 22. 04. 2019, 1 female, 1 male; Cserszegtomaj: Fagyos-kereszt, 17. 04. 2019, 1 male; Balatongyörök: Bélap-völgy, 23. 04. 2019, 2 females, 21. 04. 2019, 1 male, 22. 04. 2019, 1 male; Balatongyörök: Mogyorós út: Szőlőhegyek, 18. 04. 2019, 2 males. Frequent. Larva on Prunus spinosa.

Periclista (Periclista) albiventris (Klug, 1816): Balatongyörök: Bélap-völgy, 22. 04. 2019, 1 female, 20. 04. 2019, 1 female, 27. 04. 2019, 2 males. Sporadic. Hostplant unknown.

Stethomostus fuliginosus (Schrank, 1781) Keszthely: Szendrey telep: forest belt, 15. 05. 1992, 1 male; Zalaszántó: Alsó-nyíres, 23. 04. 2019, 1 male. Frequent. Larva on Ranunculus acris, $R$. repens and $R$. sceleratus.

Tomostethus nigritus (Fabricius, 1804): Keszthely, 29. 04. 2000, 1 male. Sporadic. Hostplant: Fraxinus excelsior.

\section{Tenthredininae}

Aglaostigma (Astochus) aucupariae (Klug, 1817): Balatongyörök: Bélap-völgy, 16. 04. 2019, 1 female, 21. 04. 2019, 2 females, 18. 04. 2019, 2 males, 22. 04. 2019, 1 male; Balatongyörök: Mogyorós út: Szőlöhegyek, 18. 04. 2019, 1 female; Cserszegtomaj: Pilikáni dolomitbánya, 30. 03. 2019, 1 male; Cserszegtomaj: Várvölgyi út: Pörkölt-hegy, 30. 03. 2019, 1 male; Vállus: Szarvas-kotrás, 07. 04. 2019, 1 male; Vállus: Kis-Lázvölgy, 17. 04. 2019, 1 male; Várvölgy: Zsidi-rét, 30. 03. 2019, 1 female; Várvölgy: Bándi-mező, 31. 03. 2019, 1 male, 16. 04. 2019, 1 male; Zalaszántó: Alsó-nyíres, 31. 03. 2019, 1 female, 1 male; Rezi, 23. 04. 1992, 1 male; Keszthely: Újmajor on Phleum pratense 16. 04. 1991, 1 female; Keszthely: Úmajor, 18. 05. 1993, 1 female; 20. 04. 2002, 1 female. Common. Larva on Galium mollugo and G. boreale.

Aglaostigma (Astochus) fulvipes (Scopoli, 1763): Balatongyörök: Bélap-völgy, 27. 04. 2019, 1 female, 1 male, 20. 04. 2019, 1 male, 21. 04. 2019, 2 males, 22. 04. 2019, 1 male, 23. 04. 2019. 2 males, 19. 04. 2019. 1 male; Balatongyörök: Mogyorós út: Szőlőhegyek, 19. 04. 2019, 1 male; Cserszegtomaj: Fagyos-kereszt, 20. 04. 2019, 1 female, 1 male; Gyenesdiás: Lőtér, 19. 04. 2019. 1 male; Rezi: Gyöngyösi csárda, 17. 04. 2019, 1 female; Várvölgy: Zsidi-rét, 17. 04. 2019, 1 male; Keszthely: Szendrey telep, Vicia spp. 28. 05. 1992, 1 female; Keszthely: Szendrey telep, 18. 05. 1992, 1 female, 01. 05. 1992, 1 male. Common. Larva on Galium mollugo and G. verum.

Macrophya (Macrophya) albicincta (Schrank, 1776): Rezi: Gyöngyösi csárda, 17. 04. 2019, 2 females, 1 male; Balatongyörök: Bélap-völgy, 19. 04. 2019, 1 female, 27. 04. 2019, 1 female; Balatongyörök: Mogyorós út: Szőlöhegyek, 19. 04. 2019, 1 male; Cserszegtomaj: Fagyos-kereszt, 20. 04. 2019, 1 male; Vonyarcvashegy: Láprét, 21. 04. 2019, 2 females; Keszthely: park 08. 05. 1991, 1 female; Keszthely: Szendrey telep: forest belt, 21. 05. 1991, 3 females; Cserszegtomaj: Keszthely Hills: Várvölgyi út, 22. 05. 1991, 1 male; Keszthely, 13. 05. 2002, 1 female, 21. 05. 2002, 1 female, 30. 05. 1992 , 1 female, 19. 05. 1992, 1 male, 12. 05. 2010, 1 female, 16. 05. 2002, 1 female. Common. Hostplants: Sambucus ebulus, S. nigra, S. racemosa, Valeriana officinalis and Viburnum opalus.

Macrophya (Macrophya) annulata (Geoffroy, 1785): Keszthely: Szendrey telep. forest belt, 30. 06. 1991, 1 female; Keszthely, 04. 05. 2000, 1 female, 12. 05. 1992, 1 female, 29. 04. 2001, 1 male; Cserszegtomaj: Dobogó, 25. 05. 2019, 2 females, 1 male; Cserszegtomaj: Fagyos-kereszt, 01. 06. 2019, 1 male; Balatongyörök: Bélap-völgy, 25. 


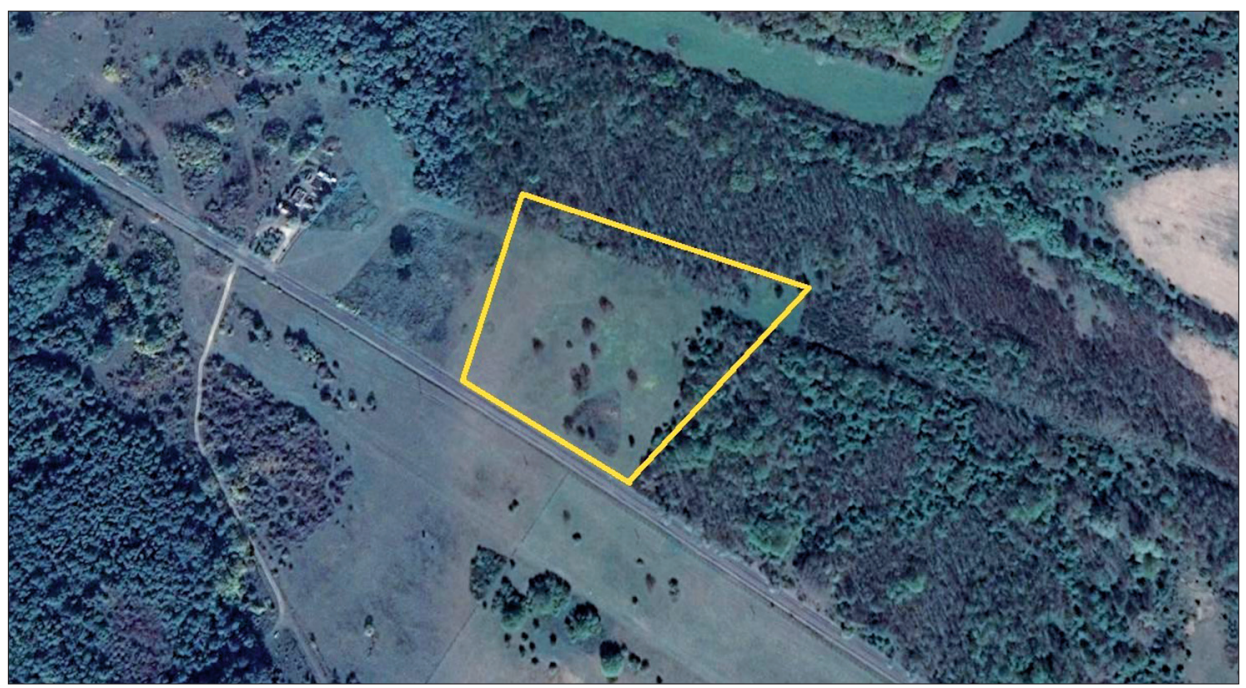

Fig. 5. Map of Várvölgy: Bándi-mező (Bándi Meadow)

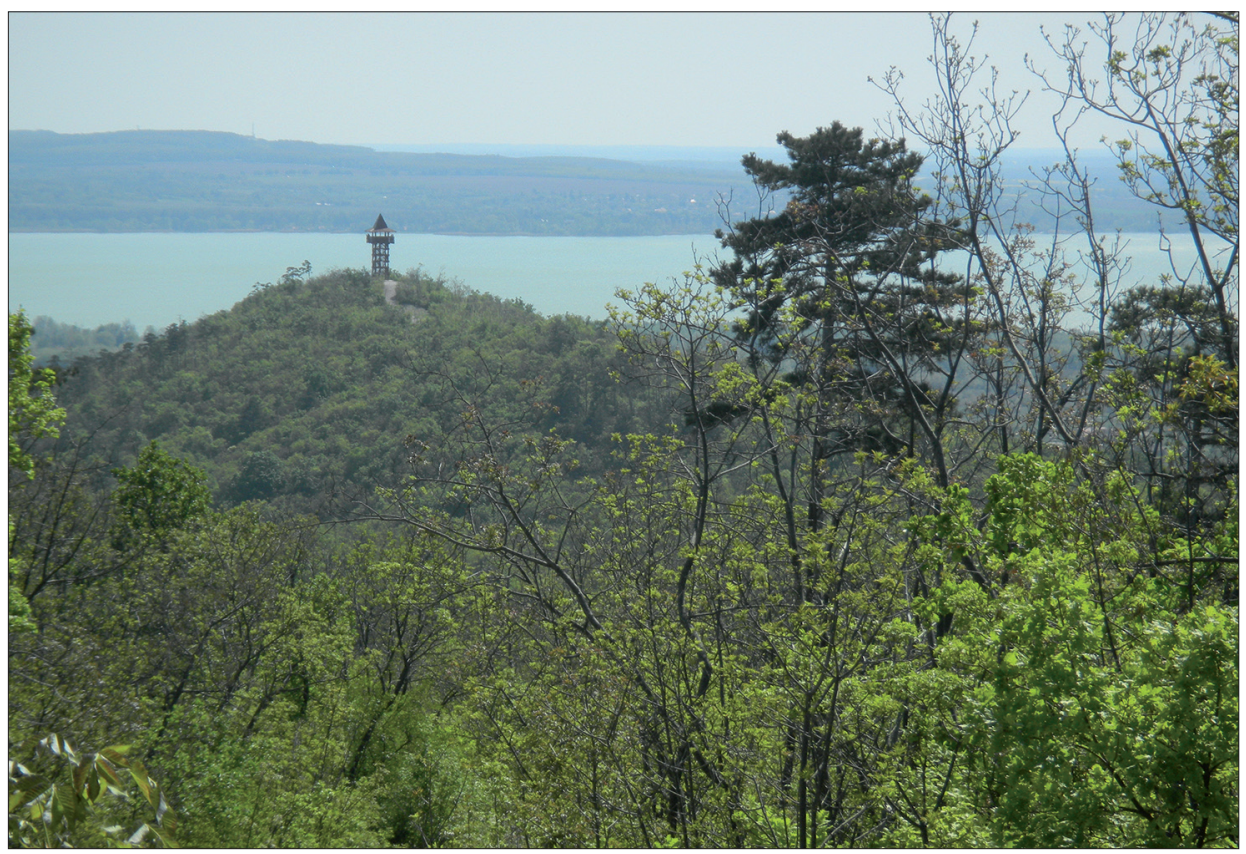

Fig. 6. Landscape of Keszthely Hills with Lake Balaton in the background

05. 2019, 1 female, 1 male, 11. 05. 2019, 1 female, 1 male, 01. 06. 2019, 1 male; Balatongyörök: Mogyorós út: Szőlöhegyek, 11. 05. 2019, 1 male. Frequent. Larva on Potentilla reptans, Origanum vulgare, Euphorbia, Rosa, Rubus and Sambucus spp. 




Fig. 7. Landscape of Bélap-völgy (Bélap Valley)

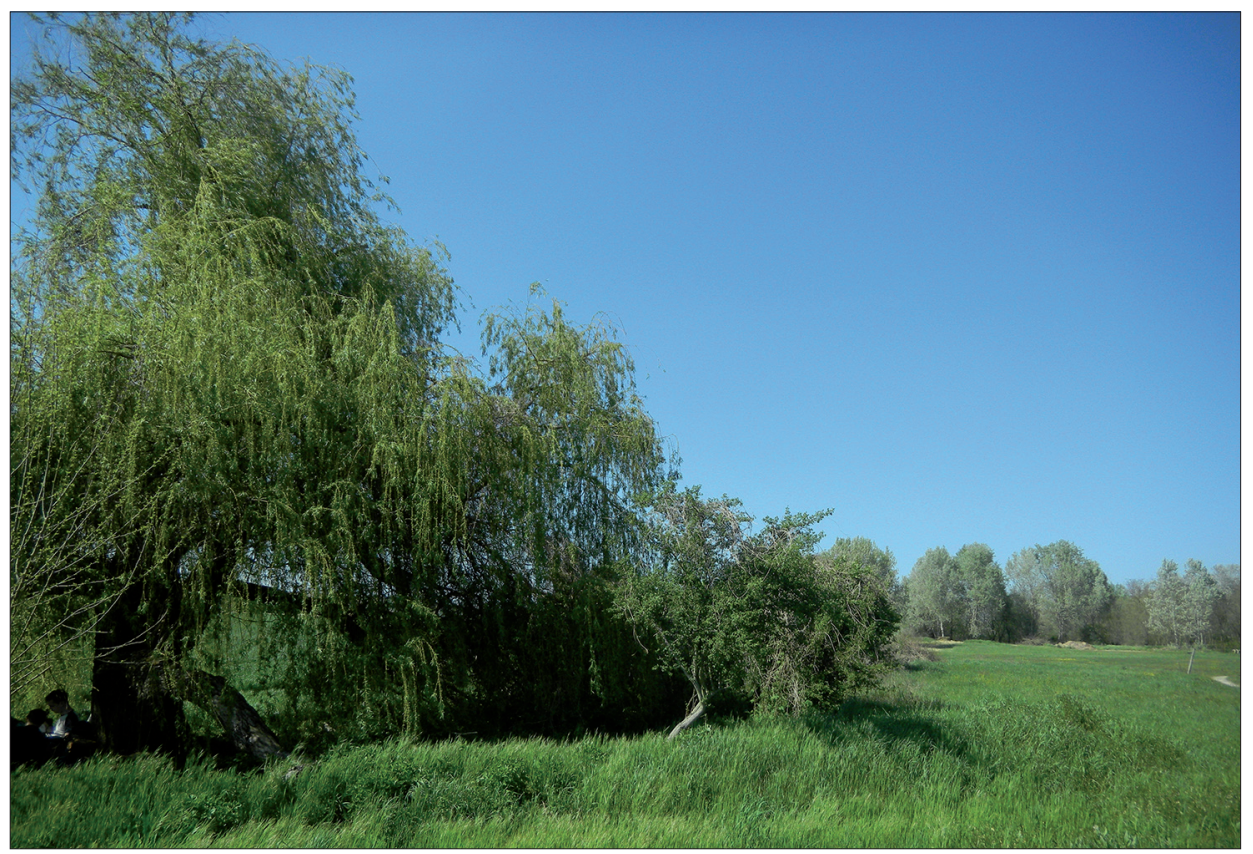

Fig. 8. Sampling site at Gyöngyösi Betyárcsárda in Rezi 
Macrophya (Macrophya) crassula (Klug, 1817): Cserszegtomaj: Dobogó, 25. 05. 2019, 1 female. Sporadic. Hostplant: Sambucus ebulus.

Macrophya (Macrophya) duodecimpunctata ssp. duodecimpunctata (Linné, 1758): Balatongyörök: Mogyorós út: Szőlöhegyek, 11. 05. 2019, 1 male; Keszthely: Szendrey telep, forest belt, 21. 05. 1991, 1 female; Keszthely, 10. 05. 1993, 1 female, 11. 05. 2000, 1 female, 22. 05. 2002, 1 female. Common. Hostplants: Graminae, Cyperaceae and Carex spp.

Macrophya (Macrophya) erythrocnema A. Costa, 1859: Cserszegtomaj: Dobogó, 25. 05. 2019, 1 female. Sporadic. Hostplant: Knautia arvensis.

Macrophya (Macrophya) montana (Scopoli, 1763): Cserszegtomaj: Dobogó, 11. 05. 2019, 2 females, 25. 05. 2019, 1 female, 1 male, 18. 05. 2019, 1 female; Balatongyörök: Bélap-völgy, 25. 05. 2019, 1 female, 1 male, 18. 05. 2019, 1 female, 01. 06. 2019, 2 females, 11. 05. 2019, 1 male; Cserszegtomaj: Várvölgyi út: Pörkölt-hegy, 01. 06. 2019, 1 female, 25. 05. 2019, 1 male; Keszthely, 24. 05. 1997, 1 female. Common. Hostplant: Rubus caesius.

Macrophya (Macrophya) postica (Brullé, 1832): Balatongyörök: Bélap-völgy, 01. 06. 2019, 2 females. Frequent. Hostplant unknown.

Macrophya (Macrophya) recognata Zombori, 1979: Rezi, 01. 08. 1983, 1 female. Sporadic. Hostplant unknown.

Macrophya (Macrophya) ribis (Schrank, 1781): Keszthely: Szendrey telep: forest belt 30. 06. 1991, 2 females; Keszthely: Szendrey telep: forest belt, 22. 06. 1991, 1 male. Frequent. Larva on Sambucus nigra.

Macrophya (Macrophya) rufipes (Linné, 1758): Cserszegtomaj: Dobogó, 11. 05. 2019, 1 female, 25. 05. 2019, 2 females, 2 males; Várvölgy: Bándi-mező, 01. 06. 2019, 1 female, 1 male. Frequent. Larva on Agrimonia eupatoria.

Macrophya (Pseudomacrophya) punctumalbum (Linné, 1767): Keszthely, 16. 05. 1992, 1 female. Sporadic. Hostplants: Fraxinus spp. and Ligustrum spp.

Pachyprotasis rapae (Linné, 1767): Keszthely: Szendrey telep: forest belt, 1991. 05. 21. 1 female; Keszthely, 01. 05. 1992, 1 male. Regularly common, frequently dominant species however in 2019, I didn't collected it. Hostplants: Solanum tuberosum, Pedicularis palustris, Angelica sylvestris, Veronica beccabunga, Betonica officinalis, Corylus avellana, Salix caprea, Fraxinus excelsior, Tussilago farfara, Symphoricarpos albus, Scrophularia, Solidago, Verbascum, Origanum, Atropa, Sarothamnus, Senecio, Polygonum, Aspidium, Epilobium, Hypericum, Galeopsis, Mentha, Polystichum, Plantago, Quercus and Stachys spp.

Rhogogaster (Rhogogaster) chlorosoma (Benson, 1943): Gyenesdiás: Kőbánya, 04. 07. 1994, 1 female.Frequent. Hostplants: Pteridium aquilinum, Alnus glutinosa, Circaea, Prunus spp., Ranunculus spp., Rosa spp., Salix alba, S. purpurea, Stellaria spp., Filipendula ulmaria, Populus tremula, Padus spp., Betula spp., Corylus avellana and Sorbus spp.

Rhogogaster (Cytisogaster) picta (Klug, 1817): Balatongyörök: Mogyorós út: Szőlöhegyek, 18. 04. 2019, 1 female. Sporadic. Cytisus scoparius, C. nigricans, Genista germanica and G. tinctoria.

Sciapteryx consobrina (Klug, 1816): Cserszegtomaj: Büdöskúti út, 07. 04. 2019, 1 female; Balatongyörök: Bélap-völgy, 20. 04. 2019, 1 female. Frequent. Larval hosts: Adoxa spp., Anemone spp. and Ranunculus ficaria.

Sciapteryx costalis (Fabricius, 1775): Cserszegtomaj: Büdöskúti út, 07. 04. 2019, 1 male; Zalaszántó: Alsó-nyíres, 31. 03. 2019, 1 male; Keszthely, 17. 05. 1992, 1 male. Frequent. Hostplant: Ranunculuis acris. 
Tenthredo (Tenthredella) atra Linné, 1758: Zalaszántó: Kovácsi-hegy, 14. 08. 1966, 1 female. Frequent. Larval hosts: Lamium, Mentha, Plantago, Vicia, Ranunculus, Scabiosa, Brassica and Solanum spp.

Tenthredo (Cephaledo) bifasciata ssp. violacea (André, 1881): Keszthely, 24. 04. 2002, 1 female. Frequent. Hostplant unknown.

Tenthredo (Cephaledo) neobesa Zombori, 1980: Vállus: Láz-tető: 28. 05. 1964, 1 female. Rare. Hostplant unknown.

Tenthredo (Cephaledo) excellens (Konow, 1886): Balatongyörök: Bélap-völgy, 23. 04. 2019, 1 female. Sporadic. Hostplant unknown.

Tenthredo (Elinora) flaveola Gmelin, 1790: Keszthely, 24. 04. 2002, 1 female. Sporadic. Brassica oleracea, B. nigra, Sinapis arvensis, S. alba, Raphanus raphanistrum and Isatis tinctoria.

Tenthredo (Endotethryx) campestris Linné, 1758: Keszthely: Szendrey telep: forest belt, 14. 05. 1991, 1 female; Keszthely, 25. 05. 2002, 1 female, 17. 05. 2009, 1 female, 05. 05. 2001, 1 male. Frequent. Hostplant: Aegopodium podagraria.

Tenthredo (Maculedo) maculata Geoffroy, 1785: Keszthely, 13. 05. 2002, 1 female, 15. 05. 2002, 1 female; Vállus: Láz-tető: 28. 05. 1964, 1 male. Sporadic. Host plants: Brachypodium spp. and Dactylis spp.

Tenthredo (Maculedo) vespiformis Schrank, 1781: Vállus: Láz-tető: 28. 05. 1964, 1 female. Rare in Hungary. Hostplant unknown.

Tenthredo (Tenthredella) livida Linné, 1758: Keszthely: Szendrey telep: forest belt on the flower of Aegopodium podagraria 30. 06. 1991, 1 female. Sporadic, locally frequent. Larva on Salix sp., Corylus avellana; Epilobium spp.; Lonicera spp.; Pteridium aquilinum; Rosa spp., Sorbus aucuparia, Symphoricarpos albus, Viburnum opulus, Arctium lappa and Athyrium filix-femina.

Tenthredo (Tenthredella) solitaria ssp. solitaria Scopoli, 1763: Vállus: Láz-tető: 28. 05. 1964, 1 female, 1 male. Sporadic in Hungary. Larva on Euphorbia cyparissius.

Tenthredo (Eurogaster) mesomela Linné, 1758: Keszthely: Szendrey telep: forest belt 1991. 05. 21. 1 female; Keszthely 29. 05., 1 female; Vállus, 28. 05. 1964, 1 female. Frequent. Larval hosts: Polygonum persicaria, Arctium lappa, Heracleum spp., Ranunculus spp., Epilobium spp., Rumex spp, Salix spp., Veronica sp., Tussilago spp., Petasites sp., Senecio sp., Solidago sp., and Stachys spp.

Tenthredo (Temuledo) temula Scopoli, 1763: Balatongyörök: Bélap-völgy, 23. 04. 2019, 1 female, 27. 04. 2019, 1 female, 2 males, 11. 05. 2019, 1 female. Frequent, locally common. Larva on Ligustrum and Origanum spp.

Tenthredo (Tenthredo) brevicornis (Konow, 1886): Zalaszántó: Kovácsi-hegy, 14. 08. 1966, 1 male. Frequent. Hostplant: Lotus corniculatus.

Tenthredo (Tenthredo) marginella Fabricius, 1793: Zalaszántó: Tátika-erdö, 13. 08. 1966, 2 females, 2 males. Sporadic. Hostplants: Mentha and Ocimum spp.

Tenthredo (Tenthredo) notha Klug, 1817: Zalaszántó: Kovácsi-hegy, 14. 08. 1966, 10 females, 9 males; Zalaszántó: Tátika-erdö, 13. 08. 1966, 4 females, 1 male. Frequent. Hostplants: Trifolium repens and Vicia cracca.

Tenthredo (Tenthredo) vespa Retzius, 1783: Zalaszántó: Kovácsi-hegy, 14. 08. 1966, 1 male; Zalaszántó: Tátika-erdő, 13. 08. 1966, 7 females, 5 males. Frequent. Hostplants: Lonicera caprifolium, Syringa vulgaris, Viburnum opulus, Fraxinus excelsior, Jasminum officinale, Rosa spp., Spiraea spp., Acer platanoides, Ligustrum vulgare, Symphoricarpos albus, S. alba.

Tenthredo (Tenthredo) zona Klug, 1817: Vállus: Láz-tető: 28. 05. 1964, 1 female. Sporadic. Hostplant: Hypericum perforatum. 
Tenthredo (Zonuledo) amoena Gravenhorst, 1807: Cserszegtomaj: Csóka-kő, 01. 08. 1983, 1 female. Frequent. Larva on Hypericum maculatum and H. perforatum.

Tenthredo (Zonuledo) distinguenda (Stein, 1885): Balatongyörök: Bélap-völgy, 11. 05. 2019, 1 male, 18. 05. 2019, 1 male; Várvölgy: Bándi-mező, 11. 05. 2019, 1 male. Frequent. Hostplant unknown.

Tenthredo (Zonuledo) zonula Klug, 1817: Balatongyörök: Bélap-völgy, 11. 05. 2019, 1 female, 2 males, 18. 05. 2019, 1 female, 25. 05. 2019, 3 females, 01. 06. 2019, 1 female, 25. 05. 2019, 1 male; Cserszegtomaj: Dobogó, 18. 05. 2019, 2 females, 11. 05. 2019, 2 males, 25. 05. 2019, 1 male; Várvölgy: Bándi-mezö, 01. 06. 2019, 1 male. Frequent. Hostplant: Hypericum perforatum.

Tenthredopsis friesei (Konow, 1884): Keszthely, 14. 05. 2002, 1 female. Frequent. Hostplants: Holcus mollis and other Poaceae.

Tenthredopsis lactiflua (Klug, 1817): Balatongyörök: Bélap-völgy, 27. 04. 2019, 1 female. Sporadic. Hostplant unknown.

Tenthredopsis litterata (Geoffroy, 1785): Cserszegtomaj: Dobogó, 11. 05. 2019, 1 male; Keszthely, 20. 05. 2002, 1 female, 11. 05. 2002, 1 male, 10. 05. 2002, 1 male. Frequent. Larva on Agrostis, Dactylis and Calamagrostis spp.

Tenthredopsis nassata (Linné, 1767): Keszthely, 25. 04. 2002, 1 female, 10. 05. 1992, 1 female. Frequent. Hostplants: Dactylis glomerata, Deschampsia caespitosa, D. calmagrostis, Flexuosa spp., Holcus spp., Lolium perenne, Agropyron spp., Carex spp., Anthriscus silvestris and Artemisia spp.

Tenthredopsis ornata (Serville, 1823): Cserszegtomaj: Dobogó, 11. 05. 2019, 1 female, 18. 05. 2019, 1 female; Keszthely, 05. 04. 1993, 1 female. Frequent. Larva on Brachypodium sylvaticum.

Tenthredopsis sordida (Klug, 1817): Balatongyörök: Bélap-völgy, 27. 04. 2019, 2 males. Frequent. Larva on Arrhenatherum elatius, Lolium perene, Carex spp., Calamagrostis spp. and Dactylis glomerata.

Tenthredopsis stigma (Fabricius, 1798): Cserszegtomaj: Dobogó, 11. 05. 2019, 5 females, 1 male, 18. 05. 2019, 2 females, 1 male; Balatongyörök: Bélap-völgy, 18. 05. 2019, 1 female; Várvölgy: Bándi-mezö, 01. 06. 2019, 1 female. Frequent. Hostplant: Triticum intermedium.

Tenthredopsis tarsata (Fabricius, 1804): Cserszegtomaj: Keszthely Hills. Side of forest road, 1992. 05. 16. 1 female; Keszthely, 13. 05. 1993, 1 female. Frequent. Hostplants: Poaceae, particularly Brachypodium silvaticum.

Tenthredopsis tessellata (Klug, 1817): Cserszegtomaj: Dobogó, 23. 04. 2019, 1 female, 22. 04. 2019, 1 female, 11. 05. 2019, 1 female, 21. 04. 2019, 1 male; Cserszegtomaj: Várvölgyi út: Pörkölt-hegy, 25. 05. 2019, 1 female; Keszthely, 10. 05. 1993, 1 female. Sporadic. Larva on Deschampsia, Dactylis, Aira and Lolium spp.

\section{Nematinae}

Cladius (Priophorus) brullei (Dahlbom, 1835): Balatongyörök: Bélap-völgy, 19. 04. 2019, 1 female; Cserszeg 21-20. 06. 1992, 1 female. Frequent. Larva on Rubus spp.

Cladius (Cladius) pectinicornis (Geoffroy, 1785): Balatongyörök: Bélap-völgy, 06. 04. 2019, 1 female, 16. 04. 2019, 1 female, 3 males, 18. 04. 2019. 3 females, 19. 04. 2019, 1 female, 1 male, 20. 04. 2019, 2 females, 1 male, 23. 04. 2019, 1 female, 1 male, 22. 04. 2019, 1 male, 11. 05. 2019, 1 male; Zalaszántó: Alsó-nyíres, 20. 04. 2019, 1 female; Vállus: Kis-Láz-völgy, 17. 04. 2019, 1 male; Rezi: Gyöngyösi csárda, 17. 04. 2019, 1 male; Keszthely 14. 08. 1991, 1 female; Cserszeg 20-21. 07. 1992, 1 female; Cserszeg 04. 07. 1992, 1 female. Frequent. Hostplant: Rubus spp. 
Euura fagi (Zaddach, 1876): Cserszegtomaj: Keszthelyi-hgys. Várvölgyi road, 22. 05. 1991, 1 female. Rare. Larva on Fagus sylvatica.

Euura bergmanni (Dahlbom, 1835): Keszthely, 23. 05. 2002, 1 female. Frequent. Hostplant: Salix.

Euura myosotidis (Fabricius, 1804): Balatongyörök: Bélap-völgy, 20. 04. 2019, 4 females, 19. 04. 2019, 2 females, 3 males, 18. 04. 2019, 1 female, 21. 04. 2019, 5 male, 23. 04. 2019, 1 male, 27. 04. 2019, 1 male, 18. 05. 2019, 1 male; Balatongyörök: Mogyorós út: Szőlöhegyek, 18. 04. 2019, 2 females, 1 male 16. 04. 2019, 1 female, 1 male; 19. 04. 2019, 3 males; Zalaszántó: Alsó-nyíres, 20. 04. 2019, 1 female, 16. 04. 2019, 1 female, 1 male; Cserszegtomaj: Dobogó, 22. 04. 2019, 1 male; Cserszegtomaj: Fagyos-kereszt, 17. 04. 2019, 1 male; 20. 04. 2019, 1 male, Keszthely 01. 10. 1991, 1 female; Keszthely 14. 09. 1991, 1 female; Keszthely 30. 09. 1991, 1 female. Common. Larval hosts: Onobrychis and Trifolium spp.

Euura tibialis (Newman, 1837): Keszthely, 19. 05. 1992, 1 female. Frequent. Larva on Robinia pseudacacia.

Euura clitellata (Serville, 1823): Cserszegtomaj: Dobogó, 23. 04. 2019, 1 female; Keszthely: Újmajor on Agrostis s. ssp. g. 25. 04. 1990, 1 female ex lárva; Keszthely: Újmajor, magfüves 26. 10. 1990, 1 female, ex lárva; Keszthely: Újmajor on Agrostis s. ssp. g. 02. 05. 1991, 1 female ex lárva; Keszthely: Újmajor on Phleum pratense 07. 05. 1991, 1 female; Keszthely: Újmajor on Festuca rubra 14. 05. 1991, 1 female; Keszthely: Újmajor on Agrostis s. ssp. g. 29. 05. 1991, 2 females; Keszthely: Úmajor, grass culture, 26. 10. 1990, 1 female ex lárva; Keszthely: Ujmajor on Agrostis s. ssp. g. 28. 05. 1990, 1 female ex lárva; Keszthely: Újmajor on Agrostis s. ssp. g. 29. 04. 1991, 1 female. Frequent. Larval hosts: Graminae, Carex and Juncus spp.

Euura fallax (Serville, 1823) known as Pachynematus xanthocarpus (Hartig, 1840): Balatongyörök: Bélap-völgy, 23. 04. 2019, 2 males, 16. 04. 2019, 1 male; Keszthely: Újmajor 29. 03. 1989, 1 female; Keszthely: Újmajor, 01. 04. 1989, 1 female; Keszthely: Újmajor on Agrostis s. ssp. g. 09. 04. 1991, 1 female; Keszthely: Újmajor on Tricum aestivum 01. 04. 1988, 1 female. Frequent. Larva on Graminae.

Hoplocampa brevis (Klug, 1816): Keszthely, 08. 04. 1968, 10 females. Insect pest of Pyrus and Malus spp.

Hoplocampa flava (Linné, 1760): Keszthely, 08. 04. 1968, 6 females. Frequent. Larva on Prunus domestica and Prunus spinosa.

Hoplocampa minuta (Christ, 1791): Rezi on flowering Prunus domestica 23. 04. 1992,

1 female. Frequent. Larva on Prunus domestica, P. armeniaca, P. instita, P. avium and P. spinosa.

Hoplocampa testudinea (Klug, 1816): Keszthely, 21. 04. 1968, 2 females, 29. 04. 1968, 1 female. Sporadic. Larva on Malus and Pyrus spp.

Nematus (Nematus) lucidus (Panzer, 1801): Balatongyörök: Bélap-völgy, 18. 04. 2019, 1 female. Sporadic, locally frequent. Larva on Crataegus and Prunus spinosa.

Pontania (Eupontania) viminalis (Linné, 1758): Vonyarcvashegy: Láprét, 21. 04. 2019, 1 male. Sporadic. Larva on Salix purpurea.

Pristiphora (Micronematus) monogyniae (Hartig, 1840): Balatongyörök: Bélap-völgy, 22. 04. 2019, 1 female, 18. 04. 2019, 1 female. Frequent. Larva on Prunus spinosa, ocasionally on $P$. domestica.

Pristiphora (Pristiphora) albitibia (A. Costa, 1859): Balatongyörök: Mogyorós út: Szölöhegyek, 20. 04. 2019, 1 female; 16. 04. 2019, 1 male. Rare. Hostplant unknown.

Pristiphora (Pristiphora) armata (C.G. Thomson, 1862): Balatongyörök: Bélapvölgy, 27. 04. 2019, 1 male. Frequent. Larva on Crataegus spp. 
Pristiphora (Oligonematus) laricis (Hartig, 1837): Vonyarcvashegy: Láprét, 21. 04. 2019, 1 female; Balatongyörök: Bélap-völgy, 27. 04. 2019, 1 female. Sporadic. Hostplants: Larix spp.

Pristiphora (Pristiphora) fausta (Hartig, 1837): Balatongyörök: Bélap-völgy, 19. 04. 2019, 1 female. Rare. Larva on Quercus spp.

Pristiphora (Pristiphora) subbifida (C. G. Thomson, 1871): Balatongyörök: Bélapvölgy, 18. 04. 2019, 1 female, 23. 04. 2019, 1 female, 21. 04. 2019, 1 female. Sporadic. Hostplants: Acer campestre, sometimes A. pseudoplatanus and A. orientale.

\section{Discussion}

The recorded 173 species is a respectable number, although the results from one year of intensive collection augmented with other material are not adequate to assess the real species richness. The most frequent species are Aglaostigma (Astochus) aucupariae (Klug, 1817), Aglaostigma (Astochus) fulvipes (Scopoli, 1763), Athalia cordata Serville, 1823, Cladius pectinicornis (Geoffroy, 1785), Empria liturata (Gmelin, 1790), Euura myosotidis (Fabricius, 1804), Macrophya (Macrophya) albicincta (Schrank, 1776) and Macrophya (Macrophya) montana (Scopoli, 1763). High number of some Dolerus and Pachynematus species is a result of collecting in agricultural fields (grass cultures).

From this region, the first recorded species is Allantus viennensis (Schrank, 1781), recorded by Mocsáry, 1900 from Keszthely. The specimen was not found, due to some lost codes of Mocsáry (Sándor Mocsáry labelled his specimen, similarly with other contemporary authors, with numeric code and the code interpretations were written in an inventory book). Probably with the code 199 (old label) in the collection of the Natural

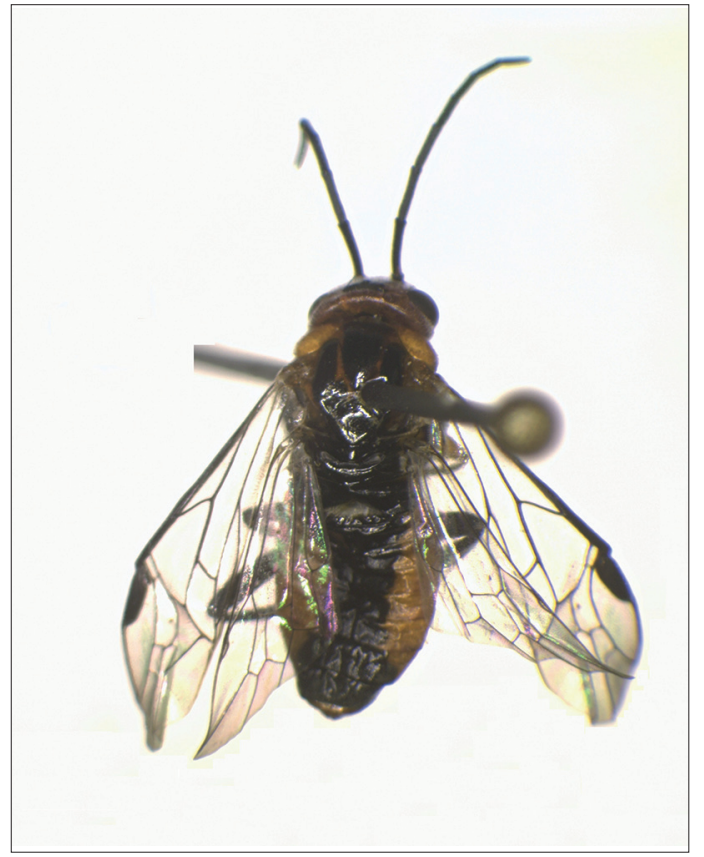

Fig. 9. Pristiphora fausta (Hartig, 1837) 
History Museum, Budapest is identical with this specimen.

Tenthredo schaefferi Klug, 1817 is deleted from the list. After the reidentification of exemplares published as T. schaefferi Kl. (Zombori, 1980), they proved to be identical with $T$. notha Klug. In interesting way, this common species was not recorded from the Keszthely Hills nor from Bakony Mts. either.

The 13 rarest species are discussed below:

Pamphilius jucundus (Eversmann, 1847): From the Carpathian Basin, this species is reported from Trencsén (Trenčín), Budapest, Nagykovácsi, Kecskemét, Lillafüred, Boksánbánya (Bocsa Montana) and the Retyezat Mountains. It is widely distributed in Europe.

Pamphilius histrio Latreille, 1812: Rare Palaearctic species with wide distribution area from Western Europe to Japan. From the Carpathian Basin, it is known from Temetvény (Považský Inovec: Hrádok), Kalocsa, Buda, Margineni, Zilah (Zalau), Drohobycz, Németbogsán (Bocsa Montana) and Mts. Cemernik: Okruglica.

Orussus unicolor Latreille, 1812: Known from Central and South Europe, and North Africa. From the Carpathian Basin, we have published records from Szádelő (Zádiel), Garamkövesd (Kamenica nad Hronom), Vállus, Budakeszi, Fényi erdő, Nagyzsuppány (Jupalnic), Budapest, Devecser: Széki, Valkó: Szent Pál-hegy, Herkulesfürdő (Baile Herculanea), Németbogsán (Bocsa Montana) and Oroszvár (Rusovce).

Tremex alchymista Mocsáry, 1886: This rare Central European species is known only from Hungary, Slovakia, Austria and Romania. It is collected in Budapest, Várpalota, Irsa, Törökkopány and Budakeszi.

Tremex magus (Fabricius, 1787): Central and Eastern European species known from Austria, Bulgaria, Czech Republic, Germany, Hungary, Poland, Romania, Russia, Slovakia and Ukraine. It occurs in the Carpathian Basin in Laxenburg, Jurský Súr, Leányfalu, Szár, and Újhely (Slovenské Nové Mesto).

Dolerus (Poodolerus) vernalis Ermolenko, 1964: Known distribution in the Carpathian Basin: Budapest: Farkasvölgy, Nagykovácsi, Nagyszeben (Sibiu-dealul Gusteritei), Keszthely and Szilvásvárad. It is recorded only in Austria, Hungary, Italy, Romania and Ukraine.

Strongylogaster mixta (Klug, 1817): From Hungary, this species is known only from Miskolc and Szenta. Other places of capture from the Carpathian Basin: Balázsvágás (Javorina), Polánahegy (Pol’ana Mt.), Nagyszeben (Hermannstadt, Sibiu), Balánbányai hgs., Retyezát (Mt. Retezat), Tiszabogdány, Brebenyeszkul (Bogdan), Croatian Karst, Trzy Korony-Pieniny, Frenštát, Trojačka, Tátralomnic (Tatranská Lomnica), Sekule (Székelyfalva), Magas Tátra: Hátsó Jávor völgy (Vysoké Tatry: Zadná Javorová dolina), Királymező (Ust-Tschorna). Palaearctic species distributed from Western Europe to Japan.

Fenella arenariae Zombori, 1978: This species known only from Hungary. Recorded occurences: Fülöpháza, Bugac, Budaörs: Naphegy, Budapest: Sváb hegy and Keszthely.

Fenella nigrita Westwood, 1839: Holarctic species, rare in Hungary. In Hungary, it is reported from Fenyőfó, Menyeke, Németbánya, Csévharaszt, Márkó, Balatonszéplak, Keszthely and Bakony: Gemence patak.

Periclista (Periclista) albiventris (Klug, 1816): In the Carpathian Basin we have records from Budapest, Simontornya, Mogyoród, Sukoró, Síkfőkút, Cserépfalu, Látrány, Mehádia, Krassó-Szörény (Caras-Severin), Mindszentkálla and Gyürüfü. Known from Croatia, Germany, Greece, Hungary, Italy, Macedonia, the Netherlands, Romania and Switzerland. 
Euura fagi (Zaddach, 1876): European species. From Hungary, it is reported from Ómassa, Börzsöny: Széna patak and Bükk: Nagyvisnyó.

Pristiphora (Pristiphora) albitibia (A. Costa, 1859): Rare species, from Hungary, it is recorded only from Budapest and Osli. European species.

Pristiphora (Pristiphora) fausta (Hartig, 1837): Only 5 specimens were collected from the Carpathian Basin: Nagyjakabfalva (Jakubov): 1 female, 15 May 1994, Malacka (Malacky): 1 female, 15 May 1993, Ihelník: 1 female, 25 Apr.-3 May 1999, Pécel: 1 female, 19th c. and Noszvaj: Síkfökút: 1 female. 13 May 1980. Distribution: Central, West and South Europe.

\section{Acknowledgement}

Author express his grateful thanks to Dr. Elöd Kondorosy (University of Pannonia Georgikon Faculty), Dr. Csaba Kutasi (Bakony Museum of Natural History) László Varga (Bakonyerdő Forestry) and István Szakács (Keszthely Forestry of Bakonyerdö) for their support of this paper.

\section{References}

AchterberG, C. 2013: Hymenoptera in Fauna Europaea version 2.6.2. http://www.faunaeur.org. last accessed 5th August 2019. Last check: 20. 09. 2019.

AchterberG, C. van \& B. van Aartsen 1986: The European Pamphiliidae (Hymenoptera: Symphyta), with special reference to The Netherlands.- Zoologische Verhandelingen Leiden 234: 1-98.

Gyurkovics, H. \& Haris, A. 2012: Sawflies (Hymenoptera: Symphyta) from Szeged and its surroundings (SE Hungary). - Natura Somogyiensis 22: 163-182.

HARIS, A. 2001: Revisional list of the Hungarian Nematinae with the description of three new species (Hymenoptera: Tenthredinidae). - Folia Entomologica Hungarica 62: 95-114.

Haris, A. 2006: Study on the Palaearctic Pristiphora species (Hymenoptera: Tenthredinidae). - Natura Somogyiensis 9: 201-277.

HARIS, A. 2009: Sawflies of the Zselic Hills, SW Hungary (Hymenoptera: Symphyta). - Natura Somogyiensis 15: $127-158$.

Haris, A. 2010: Sawflies of the Vértes Mountains (Hymenoptera: Symphyta). - Natura Somogyiensis 17: 209-238.

Haris, A. 2011: Sawflies of the Börzsöny Mountains (North Hungary) (Hymenoptera: Symphyta). - Natura Somogyiensis 19: 149-176.

HARIS, A. 2012: Sawflies of Belső-Somogy (Hymenoptera: Symphyta). - Natura Somogyiensis 22: 141-162.

Haris A. 2018a: Second contribution to the sawflies of Belső Somogy (Hymenoptera: Symphyta). - Natura Somogyiensis 31: 45-62.

Haris A. 2018b: Sawflies from Külső-Somogy, South-West Hungary (Hymenoptera: Symphyta). - Natura Somogyiensis 32: 147-164.

MocsÁry, S. 1900: Ordo Hymenoptera. p. 7-113. - In: Paszlavsky, J. (ed.): Fauna Regni Hungariae, Regia Societas Scientiarum Naturalium Hungarica, Budapest.

Prous, M.; Blank, S.; Goulet, H.; Heibo, E.; Liston, A.; Malm, T.; Nyman, T.; Schmidt, S.; Smith, D.; Vardal, H.; Viitasaari, M.; Vikberg, V. and TAeger, A. 2014: The genera of Nematinae (Hymenoptera, Tenthredinidae). - Journal of Hymenoptera Research 40: 1-69.

Roller, L. 1993: New records of sawflies (Hymenoptera: Symphyta) from Slovakia. - Entomological Problems 24(2): 81-84.

Roller, L., 1994: Faunistics records. Symphyta. - Entomological Problems 25(2): 24.

Roller, L. 1996: New records of sawflies (Hymenoptera, Tenthredinidae) in Slovakia. - Biologia, Bratislava 51(1): 549-550. 
Roller, L. 1998: Sawfly (Hymenoptera, Symphyta) community in the Devínska Kobyla National Nature Reserve. - Biologia, Bratislava 53(2): 213-221.

Roller, L. 1999a: Spoločenstvá hrubopásych (Hymenoptera: Symphyta) vybraných zoogeografických regiónov Slovenska. - PhD thesis, Ústav zoológie, Slovenská akadémia vied, Bratislava, 180 pp.

Roller, L. 1999b: First records of Nematinae (Hymenoptera, Symphyta, Tenthredinidae) in Slovakia. Biologia, Bratislava 54(5): 599-600.

RoLler, L. 1999c: Faunistic records from Slovakia. Hymenoptera: Symphyta: Tenthredinidae: Nematinae. Entomological Problems 30(1): 30.

Roller, L. 1999d: Faunistic records from Slovakia. Hymenoptera: Symphyta: Tenthredinidae: Nematinae. Entomological Problems 30(1): 52.

Roller, L. 1999e: Check list of the sawflies (Hymenoptera: Symphyta) of Slovakia. - Entomological Problems 30(2): 37-48.

Roller, L., 2000a Zubačkovité (Megalodontesidae) - hrubopáse xerotermov. - Hmyz 1(1): 17-18.

Roller, L., 2000b: First records of Blasticotomidae, Tenthredinidae, Pamphiliidae (Hymenoptera) from Slovakia. - Biologia, Bratislava 55(5): 561-562.

Roller, L., 2000c: Súčasný stav poznania fauny hrubopásych (Hymenoptera, Symphyta) na Slovensku. Správy Slovenskej zoologickej spoločnosti 18: 109-114.

Roller, L., 2001: Príspevok k poznaniu hrubopásych (Hymenoptera, Symphyta) a rohačkovitých (Diptera, Sciomyzidae) prírodnej pamiatky Mitická slatina. p. 32-36. - In: MáJSKY, J. (ed.), Zborník výsledkov inventarizačného výskumu prírodnej pamiatky Mitická slatina. Občianske združenie Pre Prírodu, Trenčín, $99 \mathrm{pp}$.

Roller, L. 2004. Hrubopáse blanokrídlovce (Hymenoptera, Symphyta) Tematínskych kopcov. - Entomofauna Carpathica 16: 56-64.

Roller, L. 2005: Blanokrídlovce (Hymenoptera): hrubopáse (Symphyta). pp. 117-123. - In: Fauna Devínskej Kobyly. APOP, Bratislava, $181 \mathrm{pp}$.

Roller, L. 2006a: Seasonal flight activity of sawflies (Hymenoptera, Symphyta) in submontane region of the West Carpathians, Central Slovakia. - Biologia, Bratislava 61(2): 193-205.

Roller, L. 2006b: Hrubopáse blanokrídlovce (Hymenoptera, Symphyta) Tematínskych vrchov - zhrnutie faunistického výskumu. p. 53-55. - In: K. RAJCová (ed.): Najvzácnejšie prírodné hodnoty Tematínskych vrchov. Zborník výsledkov inventarizačného výskumu územia európskeho významu Tematínske vrchy. KOZA, Trenčín a Pre Prírodu, Trenčín, 101 pp.

Roller, L. 2010. Hrubopáse blanokrídlovce (Hymenoptera: Symphyta) PR Šúr, pp. 215-235. - In: MaJZLAN, O., VIDLIČKA, L. (eds). Príroda rezervácie Súr. Ústav zoológie SAV, Bratislava, 410 pp.

Roller, L. Lukáš, J., 1999: New records of sawflies (Hymenoptera, Symphyta) in Slovakia. - Biologia, Bratislava 54(2): 225-228.

Roller L. Beneš K., Blank S. M., Holuša J., Jansen E., Jänicke M., Kaluza S., Kehl A., Kehr I., Kraus M., Liston A. D., Nyman T., Nie H., Savina H., Taeger A., Wei M. 2006: Contribution to the knowledge of sawfly fauna (Hymenoptera, Symphyta) of the Low Tatras National Park in Central Slovakia. - Naturae Tutela 10: 57-72.

Roller, L. \& MaceK, J. 2017: Provnález Hrubopásych Blanokrídlovcov (Hymenoptera, Symphyta) na Slovensku - Entomofauna carpathica, 29(1): 53-63.

Roller, L. \& Olšovský, T. 2012: Prvonálezy hrubopásych blanokrídlovcov (Hymenoptera, Symphyta) v slatinných lesoch s tavol'níkom vŕbolistým (Spiraea salicifolia) v Borskej nížine. - Entomofauna carpathica 24(1): 15-20.

Zhelochovtsev, A. N. 1988: Otryad Hymenoptera - Pereponchatokrylye, Podotryad Symphyta Sidyachebryukhie, 7-234. In: Medvedev, K.H. (ed.) Opredelitel nasekomykh evropeiskoi chasti SSSR, Vol. 3 Hymenoptera, Part 6, Nauka, Leningrad.

Zombori, L. 1973: A Bakonyi Természettudományi Múzeum levéldarázs-gyüjteménye (Hymenoptera: Symphyta) I. - A Veszprém megyei múzeumok közleményei 12: 467-475.

Zombori, L. 1979: A Bakonyi Természettudományi Múzeum levéldarázs-gyűjteménye (Hymenoptera: Symphyta) II. - A Veszprém megyei múzeumok közleményei 14: 211-220.

Zombori, L. 1980: A Bakonyi Természettudományi Múzeum levéldarázs-gyüjteménye (Hymenoptera: Symphyta) III. - A Veszprém megyei múzeumok közleményei 15: 181-188.

ZomвoRI, L. 2016: Levéldarázs-alkatúak IV. Tenthredinoidea IV. In Fauna Hungariae 9. Hymenoptera I. Fauna Hungariae 174. 3/c booklet. - Mondat Kft. and Hungarian Natural History Museum, Budapest 160 pp. 